h

\section{INGENIERÍA SÍSMICA}

Revista de Ingeniería Sísmica

ISSN: 0185-092X

javiles@tlaloc.imta.mx

Sociedad Mexicana de Ingeniería Sísmica

México

Tena Colunga, Arturo; Juárez Ángeles, Artemio; Salinas Vallejo, Víctor Hugo Resistencia y deformación de muros de mampostería combinada y confinada sujetos a cargas laterales

Revista de Ingeniería Sísmica, núm. 76, enero-junio, 2007, pp. 29-60

Sociedad Mexicana de Ingeniería Sísmica

Distrito Federal, México

Disponible en: http://www.redalyc.org/articulo.oa?id=61807603

Cómo citar el artículo

- Número completo

- Más información del artículo

- Página de la revista en redalyc.org

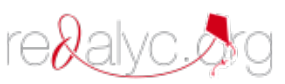

Sistema de Información Científica

Red de Revistas Científicas de América Latina, el Caribe, España y Portugal Proyecto académico sin fines de lucro, desarrollado bajo la iniciativa de acceso abierto 


\title{
RESISTENCIA Y DEFORMACIÓN DE MUROS DE MAMPOSTERÍA COMBINADA Y CONFINADA SUJETOS A CARGAS LATERALES
}

\author{
Arturo Tena Colunga ${ }^{1}$ Artemio Juárez Ángeles ${ }^{2}$ y Víctor Hugo Salinas Vallejo ${ }^{2}$
}

\begin{abstract}
RESUMEN
Se presentan los resultados más relevantes del ensaye cíclico de dos muros de mampostería combinada y confinada. Los ensayes se realizaron conforme al protocolo para ensayes cíclicos establecido en el Apéndice A de las Normas Técnicas para Diseño y Construcción de Estructuras de Mampostería (NTCM-2004), por lo que los especimenes se sujetaron a ciclos repetidos, primero ante cargas dadas (hasta la carga de agrietamiento), y después a deformaciones (distorsiones angulares) impuestas de manera creciente. Se valoran tanto los mecanismos resistentes, como las características de deformación de esta modalidad de mampostería, definiendo parámetros útiles para fines de análisis y diseño.
\end{abstract}

\begin{abstract}
The results of the first cycling testing conducted for two combined and confined masonry walls are reported in this paper. The cyclic testing followed the protocol established by Mexican guidelines for masonry structures (NTCM-2004), which is similar to that used worldwide for the cyclic testing of wall structures. The research not only evaluates resisting mechanisms and deformation characteristics of such walls, but also defines values of useful parameters for analysis and design.
\end{abstract}

\section{INTRODUCCIÓN}

La demanda de viviendas en México, sobre todo en las zonas conurbanas de algunas ciudades, obliga a recurrir a nuevos sistemas constructivos que permitan un ahorro, tanto en costos de materiales como en tiempos de ejecución. Uno de los nuevos sistemas empleados es mediante bloques de concreto ligero y tabiques de barro recocido, alternados en hiladas, el cual se define como mampostería combinada (figura 1). Este tipo de mampostería, que cae dentro de la confinada, permite un ahorro en costos y tiempos de ejecución, además de tener una apariencia agradable o estética.

Esta modalidad es de origen autóctono y tiene sus antecedentes en algunas construcciones coloniales donde se alternaban piedras naturales con ladrillos, como por ejemplo, el edificio que se muestra en la figura 2 y que forma parte del centro histórico de la ciudad de Mérida, Yucatán. Al parecer, esta modalidad se populariza en tiempos recientes principalmente por la iniciativa e inquietud de los habitantes de los estados de Puebla, Tlaxcala, Hidalgo, Oaxaca y Estado de México, quienes intentaron

Artículo recibido el 2 de enero de 2007 y aprobado para su publicación el 9 de marzo de 2007. Se aceptarán comentarios y/o discusiones hasta cinco meses después de su publicación.

1 Profesor, Departamento de Materiales, Universidad Autónoma Metropolitana Azcapotzalco, Av. San Pablo 180, Col. Reynosa Tamaulipas, 02200 México, DF, e-mail: atc@correo.azc.uam.mx

2 Estudiante de Maestría en Estructuras, Universidad Autónoma Metropolitana, Av. San Pablo 180, Col. Reynosa Tamaulipas, 02200 México,DF, e-mail: arjuan_uam@hotmail.com, vicusalva@hotmail.com 
resolver con esta modalidad, de manera económica, el problema de agrietamiento que presentan los muros de bloques, por lo que decidieron alternarlos con ladrillos rojos para evitar la propagación de grietas en los bloques de concreto, funcionando satisfactoriamente para el caso de asentamientos (Salinas 2005, Juárez 2007). El uso de este tipo de mampostería data de los 80, de acuerdo con la información recabada de los habitantes de estas construcciones, teniendo auge en la década de los 90 (Juárez 2007). Dicha modalidad comienza a utilizarse también en el Distrito Federal y de manera predominante en otros estados de la República Mexicana, como Querétaro y Veracruz.

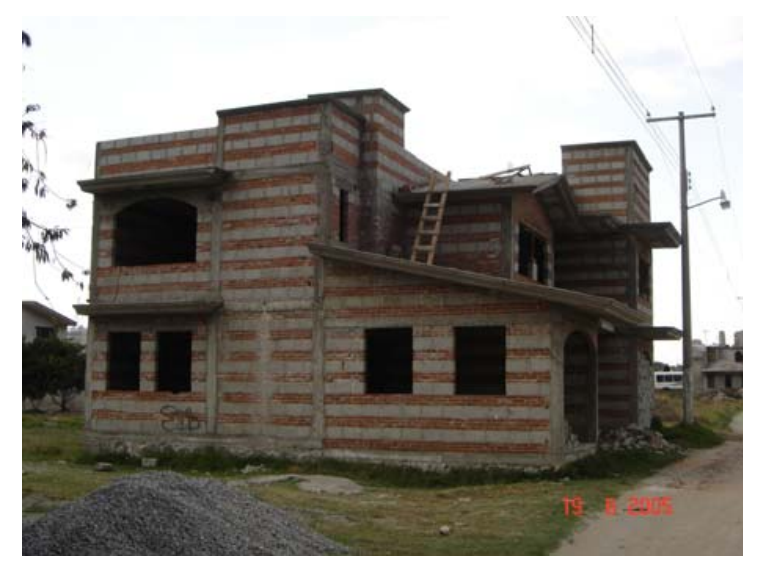

Figura 1. Mampostería combinada y confinada

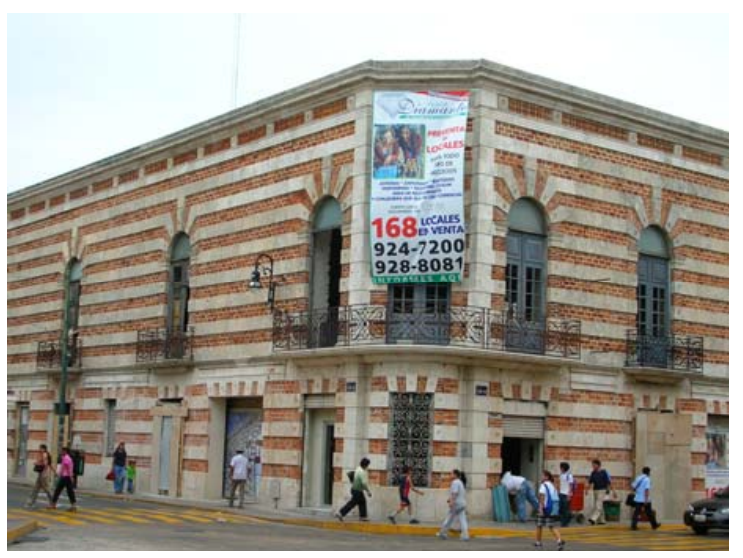

Figura 2. Estructura colonial en Mérida

Existen variantes en el uso de esta mampostería, por lo que se pueden distinguir diferentes configuraciones en el arreglo de las hiladas y en el uso que se le está dando. La configuración más común observada en los lugares donde se está empleando se muestra en la figura 1, en la que se colocan tres hiladas de tabique de barro recocido por una hilada de bloque de concreto, debido en gran medida a la comodidad que el obrero tiene al pegar la mampostería, ya que la dimensión en altura de las hiladas de tabique corresponden aproximadamente a la altura del bloque. Entre las variantes se encuentran desde configuraciones donde se alterna una hilada de tabique con una hilada de bloque (figura 3a), hasta casos donde se alternan tres hiladas de bloque con tres de ladrillo. En la figura 3 se muestran las variantes más frecuentes en la configuración de la mampostería combinada. En algunos casos varía también el color del bloque, lo que les brinda una mejor apariencia estética (Juárez 2007).

Una variante excepcional que se encontró en Cuautitlán, Estado de México, se ilustra en la figura 4 (Juárez 2007), donde la mampostería se combina en un patrón de liga que en la literatura mundial se conoce como "coursed Ashlar" (Schneider y Dickey 1993), liga utilizada en muros bloques de concreto cuyo antecedente data de construcciones medievales con muros de piedra natural de la Gran Bretaña. Finalmente, en tiempos recientes, se está combinando también al ladrillo rojo recocido con el adobe estabilizado, principalmente para construcciones residenciales, como se ilustra en la figura 5 (Juárez 2007).

\section{ANTECEDENTES EXPERIMENTALES}

La investigación experimental previa que se ha hecho en México en mampostería no contempla a la mampostería combinada descrita anteriormente, por lo que surge la necesidad de estudiarla, para valorar sus mecanismos de resistencia y deformación ante la eventual acción de sismos, ya que se está utilizando en zonas de sismicidad importante de México. 


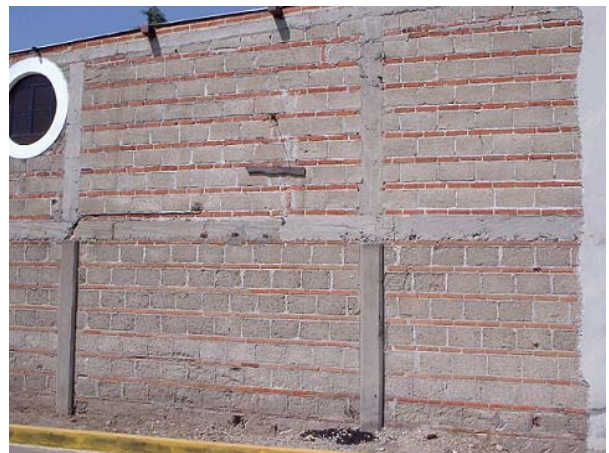

a) Una hilada de bloque por una de ladrillo

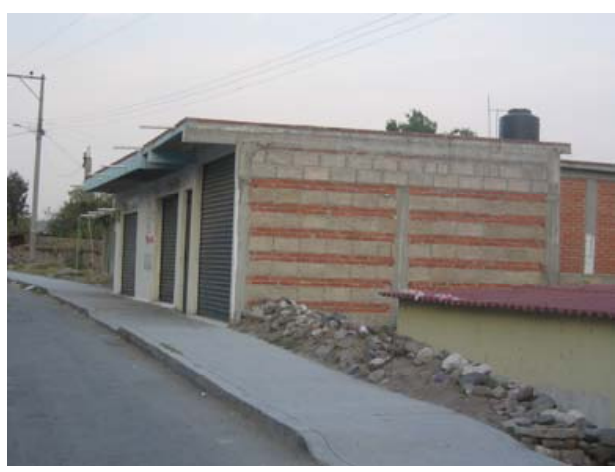

c) Una hilada de bloque por dos de ladrillo

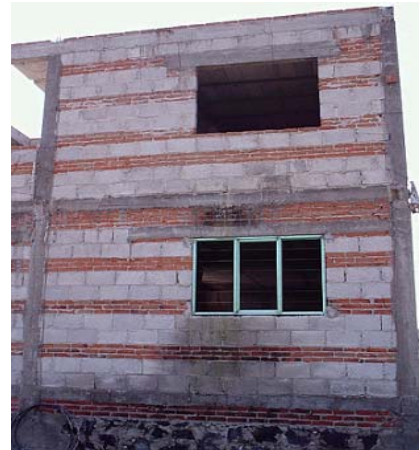

b) Dos hiladas de bloque por tres de ladrillo

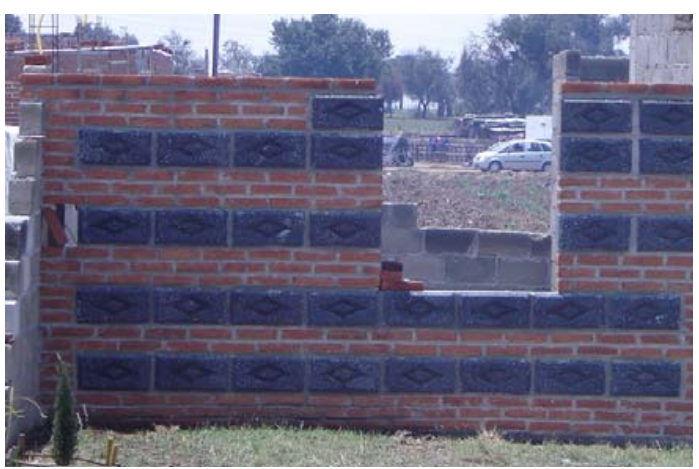

d) Uso de bloque de color diferente (negro)

Figura 3. Variantes de mampostería combinada

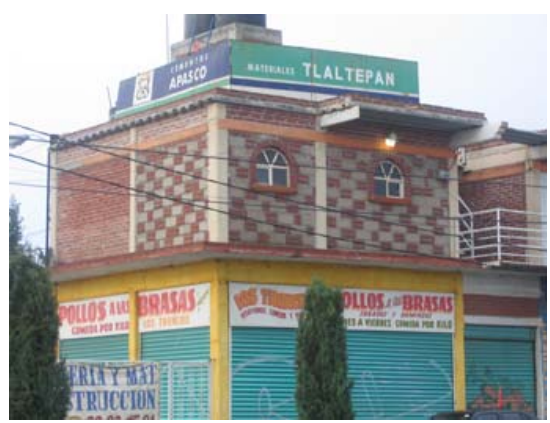

Figura 4. Mampostería combinada en arreglo "coursed Ashlar”

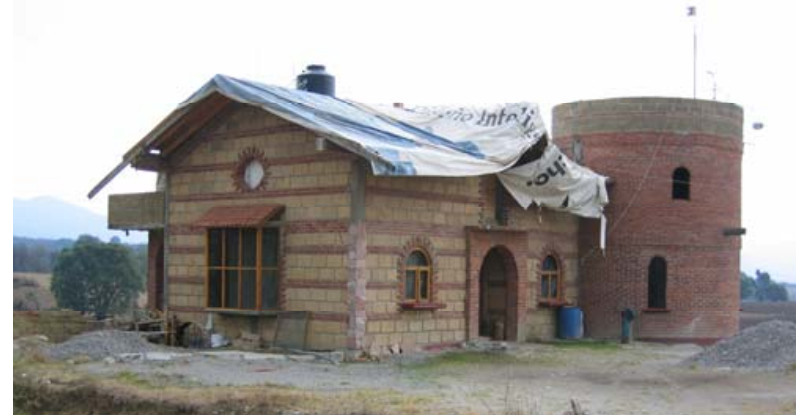

Figura 5. Mampostería combinada con adobe estabilizado y ladrillo rojo recocido

Este trabajo presenta los resultados más relevantes del ensaye cíclico de dos muros de mampostería combinada y confinada con la configuración de aparejo más utilizada (figura 1). Los ensayes se realizaron conforme al protocolo para ensayes cíclicos establecido en el Apéndice A de las Normas Técnicas para Diseño y Construcción de Estructuras de Mampostería (NTCM-2004), por lo que los especimenes se sujetaron a ciclos repetidos, primero ante cargas dadas (hasta la carga de agrietamiento), y después a deformaciones (distorsiones angulares) dadas de manera creciente. Los resultados experimentales se reportan con detalle en Salinas (2005) y Juárez (2007) y se resumen en las secciones siguientes. 


\section{MAMPOSTERÍA COMBINADA Y CONFINADA}

La mampostería combinada y confinada empleada actualmente se fabrica principalmente con piezas de barro recocido (ladrillos) y bloques sólidos de concreto ligero, los cuales se pegan mediante mortero con base en cemento, cal y arena con un espesor de junta entre 1 y $2 \mathrm{~cm}$.

Los ladrillos de barro recocido que se utilizan son piezas fabricadas artesanalmente por gente de la región (figura 6). Los tabiques artesanales producidos en la zona de Puebla y Cholula son extensamente usados en los estados de Puebla, Tlaxcala y parte de la ciudad de México y los municipios aledaños a ésta que pertenecen al Estado de México.

Los bloques de concreto ligero son manufacturados de forma mecanizada mediante vibración de una mezcla seca embebida en moldes de metal; los bloques se retiran del molde prácticamente enseguida y se curan a la intemperie. De hecho, no cuentan con un sistema de control de calidad. La mezcla está constituida con base en arena y tepojal, el cual es un agregado de origen volcánico que es ligero, poroso y de baja resistencia. Existe una gran variedad de este tipo de pieza en cuanto a color, forma y textura, aunque las piezas más utilizadas para la fabricación de muros de mampostería combinada son las que se muestran en la figura 7.

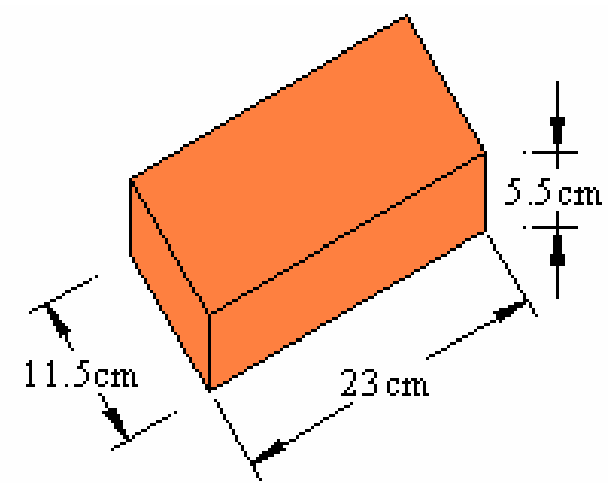

Figura 6. Ladrillo de barro rojo recocido

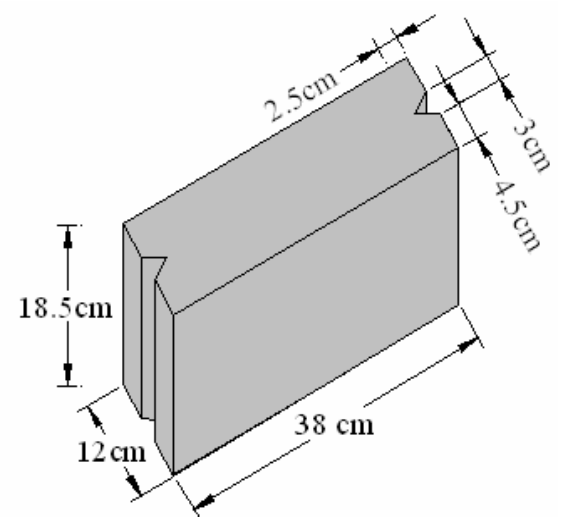

Figura 7. Bloque con saque en las juntas verticales

\section{PROPIEDADES ÍNDICE DE PIEZAS, PILAS Y MURETES}

Dada la ausencia de información experimental sobre esta modalidad de mampostería, se procedió a realizar todo el protocolo experimental con la finalidad de definir las propiedades índices del mortero, piezas, pilas y muretes de la mampostería combinada y que corresponden específicamente a las piezas y mortero cuyo tipo y características se emplearon en la fabricación de los muros de prueba. Los resultados de dichas pruebas se reportan con detalle en Salinas (2005) y Juárez (2007), y se resumen a continuación.

\section{Propiedades de las piezas}

Las dimensiones, requisitos y propiedades de las piezas, se obtuvieron experimentalmente conforme a los lineamientos establecidos por las normas mexicanas correspondientes (NMX-C-404-1997ONNCCE, NMX-C-036 y NTCM-2004). Para el caso del coeficiente de saturación y el módulo de ruptura, se tomó como referencia la norma internacional ASTM C67-91. Las propiedades obtenidas para los ladrillos y los bloques se resumen en la tabla 1, se reportan con detalle en Juárez (2007) y se describen 
en Tena (1994). Se aprecia que, en términos generales, los ladrillos tienen propiedades bastante razonables para el medio mexicano y cumplen los requisitos mínimos de las normas mexicanas correspondientes, salvo que poseen una absorción inicial muy alta que indica la necesidad de humedecer las piezas antes de su colocación. En cambio, las propiedades de los bloques son malas (pobres) en general, y no satisfacen los requisitos de las normas mexicanas, salvo el módulo de ruptura, el cual es, afortunadamente para esta modalidad, relativamente alto $\left(f_{r} \cong 0.17 \bar{f}_{p}\right)$, mayor al valor mínimo de $5 \mathrm{~kg} / \mathrm{cm}^{2}$ exigido por la norma NMX-C-404-1997-ONNCCE.

Tabla 1. Propiedades índices de las piezas

\begin{tabular}{lcc}
\hline \multicolumn{1}{c}{ Propiedad } & Ladrillo & Bloque \\
\hline Dimensiones: alto, ancho y largo $(\mathrm{cm})$ & $5.5 \times 11.5 \times 23$ & $18 \times 12 \times 38$ \\
Peso volumétrico $\gamma\left(\right.$ ton $\left./ \mathrm{m}^{3}\right)$ & 1.58 & 1.09 \\
Absorción promedio & $17.8 \%$ & $25.2 \%$ \\
Absorción inicial promedio (gramos/min) & 64.2 & 32.7 \\
Coeficiente de saturación promedio & 0.98 & 0.98 \\
Módulo de ruptura promedio, $f_{r}\left(\mathrm{~kg} / \mathrm{cm}^{2}\right)$ & 7.7 & 8.1 \\
Resistencia a la compresión & & \\
$\bar{f}_{p}\left(\mathrm{~kg} / \mathrm{cm}^{2}\right)$ & 113.8 & 47.9 \\
$C_{p}$ & 0.07 & 0.20 \\
$f^{*}{ }_{p}\left(\mathrm{~kg} / \mathrm{cm}^{2}\right)$ & $61 *$ & $27.3 *$ \\
\hline En el cálculo se empleó el valor mínimo de $\mathrm{c}_{\mathrm{p}}$ establecido en las $\mathrm{NTCM-2004}$
\end{tabular}

\section{Propiedades del mortero de pega}

El mortero empleado en la construcción de muros, muretes y pilas fue tipo I según las NTCM-2004 y se proporcionó, por tanto, conforme a las recomendaciones de la tabla 2.2 de dicha norma. La proporción en volumen usada de cemento, cal y arena fue 1:1/4:31/2. Cabe señalar que ningún tipo de mortero usado para autoconstrucción se apega a este proporcionamiento. En ocasiones, se hace caso omiso de las recomendaciones de las normas por parte de la misma supervisión. La proporción en volumen que se usa en obras de autoconstrucción es aproximadamente 1/2:1:6, cemento, cal y arena respectivamente (Juárez 2007), debido a que no hay una supervisión en el mortero que se hace en obra, ya sea para mampostería de uso estructural o no. Los albañiles que trabajan en obras sin adecuada supervisión usan la dosificación anteriormente descrita, ya que les permite trabajar sin dificultades para pegar la piezas y repellar los muros e incluso para rellenar los huecos (mampostería reforzada interiormente) y, según ellos, les ha dado buenos resultados (Juárez 2007).

La resistencia a compresión del mortero de pega se determinó de acuerdo con el ensaye especificado en la norma NMX-C-061-ONNCCE. Se elaboraron seis cubos de mortero de una muestra obtenida de cada bacha utilizada para fabricar las probetas. Se almacenaron y curaron en las mismas condiciones que las probetas a las cuales se les asociaron. El ensaye de los cubos se hizo a los 28 días aproximadamente.

Se elaboraron cubos para seis diferentes bachas de mortero elaboradas con el proporcionamiento anterior: dos para los arreglos diferentes de pilas ensayadas a compresión, dos para los arreglos de muretes ensayados a compresión diagonal y dos más para los muros ensayados a carga lateral. Del pesaje de 30 cubos se determinó que el peso volumétrico promedio del mortero es $\gamma_{j}=1.57 \mathrm{ton} / \mathrm{m}^{3}$. Del ensaye de 30 cubos de mortero a la compresión, se obtuvo que su resistencia promedio es $\bar{f}_{j}=245.7 \mathrm{~kg} / \mathrm{cm}^{2}$, su 
coeficiente de variación $c_{j}=0.319$ y su resistencia índice de diseño $f_{j}^{*}=136.6 \mathrm{~kg} / \mathrm{cm}^{2}$; ésta última superior a los $125 \mathrm{~kg} / \mathrm{cm}^{2}$ que establecen las NTCM-2004 para un mortero tipo I.

\section{Propiedades del ensaye de pilas}

La resistencia de diseño a la compresión de la mampostería combinada sobre el área bruta $\left(f^{*}{ }_{m}\right)$ y el módulo de elasticidad $\left(E_{m}\right)$, se determinaron de acuerdo con las NTCM-2004 y al proyecto de Norma Mexicana PROYNMX-S/N-ONNCCE-2005, que especifican un mínimo de nueve pilas. En este estudio se fabricaron un total de 18 pilas de acuerdo con los arreglos mostrados en la figura 8.

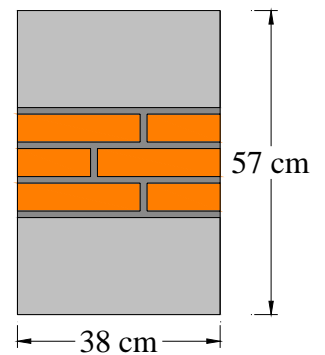

a) Pilas con arreglo 1

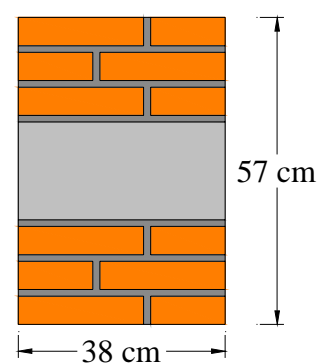

b) Pilas con arreglo 2

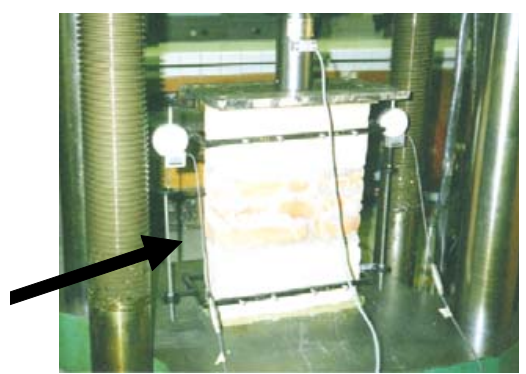

c) Pila con arreglo 1 lista para el ensaye

Figura 8. Dimensiones y configuración de las pilas para el ensaye a compresión

Se decidió construir estos dos arreglos y ensayar el doble de lo mínimo que piden las NTCM-2004, tomando en cuenta la forma en que se apareja la mampostería combinada (figura 1) y cómo podrían obtenerse resultados representativos a partir del acomodo de los ladrillos y los bloques de concreto en la pila. Resulta claro que para respetar las relaciones de esbeltez recomendadas por las normas, se tendrían como opciones aparejos de pilas donde existiera un mayor volumen de bloques (arreglo 1, figura 8a) o aparejos donde existiera un mayor volumen de ladrillos (arreglo 2, figura 8b). Dadas las notables diferencias en las propiedades mecánicas de ladrillos y bloques (tabla 1), se consideró que para obtener resultados más confiables en el estudio, debían ensayarse ambas modalidades y comparar sus resultados independientes entre sí. La relación altura espesor de todas las pilas fue de 4.78, que se encuentra dentro del intervalo que establecen las NTCM-2004 (2 a 5).

Los resultados de los ensayes realizados conforme a las normas correspondientes anteriormente citadas se presentan en Salinas (2005); se reportan con lujo de detalle en Juárez (2007) y se resumen en las tablas 2 y 3 . De estas tablas se observa que en el arreglo 1, se obtienen resistencias a la compresión, módulos de elasticidad y coeficientes de variación ligeramente mayores que con el arreglo 2 que, para fines prácticos, pueden ser poco significativas. Por ejemplo, la resistencia promedio a la compresión del arreglo 1 fue 19.4\% mayor que para el arreglo 2, mientras que la diferencia para la propiedad índice $f^{*}{ }_{m}$ fue del 22.7\%. La diferencia en el módulo de elasticidad promedio entre ambos arreglos fue sólo del 5.3\%. En vista de estos resultados, se considera válido tomar como representativos los valores ponderados de ambos arreglos, por lo que la resistencia a la compresión índice debiera tomarse como $f^{*}{ }_{m}=19.7 \mathrm{~kg} / \mathrm{cm}^{2}$ y el módulo de elasticidad promedio $E_{m}=12,244.6 \mathrm{~kg} / \mathrm{cm}^{2}$ o, en términos de la resistencia índice, $E_{m}=621.6 f_{m}^{*}$, que está bastante cercano al valor $E_{m}=600 f_{m}^{*}$ propuesto por las NTCM-2004 para mampostería de tabique de barro y otras piezas para cargas de corta duración.

Confirmando la teoría clásica de falla de pilas de mampostería a la compresión (por ejemplo, Mc Nary y Abrams 1985, Tena 1994), en ambos arreglos de las pilas la falla más común que se presentó fue con la aparición de grietas verticales generada por tracción lateral en los costados, comenzando en el 
material más resistente, que en este caso era el tabique, extendiéndose al mortero y en algunas ocasiones hasta los bloques de concreto, como se ilustra en las figuras 9a y 9b. Cabe señalar que también se presentaron fallas por aplastamiento en la que había concentración de esfuerzos en los extremos, ya sea del bloque en el caso del arreglo 1 (figura 9c) y del tabique en el arreglo 2 (figura 9d), debido a un mal acomodo de las pilas a la hora del ensaye.

Tabla 2. Resistencia de pilas a la compresión simple

\begin{tabular}{cccc}
\hline Arreglo & $\begin{array}{c}\text { Resistencia media } \\
f_{m}\left(\mathrm{~kg} / \mathrm{cm}^{2}\right)\end{array}$ & $c_{m}$ & $\begin{array}{c}f^{*}{ }^{*} \\
\mathrm{~kg} / \mathrm{cm}^{2}\end{array}$ \\
\hline 1 & 31.4 & 0.15 & 22.7 \\
2 & 26.3 & 0.17 & 18.5 \\
1 y 2 & 28.7 & 0.18 & 19.7 \\
\hline
\end{tabular}

Tabla 3. Módulo de elasticidad

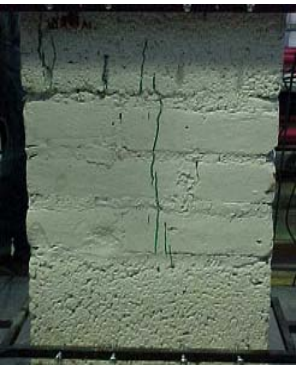

a) Pila con arreglo 1

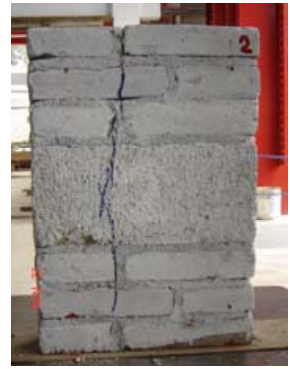

b) Pila con arreglo 2

Fallas por tracción (tensión) lateral

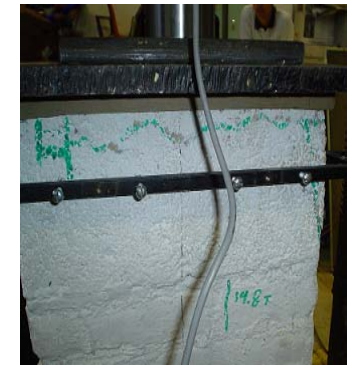

c) Pila con arreglo 1

\begin{tabular}{ccc} 
Arreglo & $\begin{array}{c}E_{m} \text { promedio } \\
\mathrm{kg} / \mathrm{cm}^{2}\end{array}$ & $C_{v}$ \\
\cline { 1 - 2 } 1 & $12,578.2$ & 0.22 \\
2 & $11,944.3$ & 0.23 \\
1 y 2 & $12,244.6$ & 0.22 \\
\hline
\end{tabular}

Fallas por aplastamiento

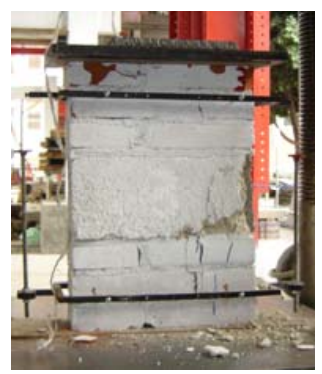

d) Pila con arreglo 2

Figura 9. Fallas observadas en las pilas durante los ensayes a compresión

\section{Propiedades del ensaye de muretes}

La resistencia de diseño a cortante o tensión diagonal de la mampostería combinada sobre el área bruta $\left(v_{m}^{*}\right)$ y el módulo de cortante $\left(G_{m}\right)$ se determinaron de acuerdo con las NTCM-2004 y al proyecto de Norma Mexicana PROYNMX-S/N-ONNCCE-2005, que especifican un mínimo de nueve muretes. En este estudio, se fabricaron un total de 18 pilas de acuerdo con los arreglos y dimensiones que se muestran en la figura 10. El diseño de los aparejos de los arreglos responden a la misma lógica que para el ensaye de pilas; determinar si existen diferencias notables en los valores indicativos y modos de falla, dado que se alternan dos materiales (ladrillos y bloques) en algunas hiladas.

Se determinó el valor de esfuerzo cortante resistente a partir del ensaye de muretes con una longitud de al menos una vez y media la máxima dimensión de la pieza y con el número de hiladas necesario para que la altura sea aproximadamente igual a la longitud (figura 10). Los muretes se ensayaron sometiéndolos a una carga de compresión a lo largo de su diagonal y el esfuerzo cortante medio se determinó dividiendo la carga máxima entre el área bruta del murete medida sobre la misma diagonal (figura 10).

Los resultados de los ensayes realizados conforme a las normas de referencia se reportan con mayor detalle en Juárez (2007) y se resumen en las tablas 4 y 5 . De los resultados presentados en estas tablas, se observa que, a diferencia de lo que sucedió en las pilas a compresión, el arreglo del murete es muy importante en la determinación de la resistencia a tensión diagonal $v^{*}$, pues existen enormes diferencias entre los resultados obtenidos para el arreglo 1 y el arreglo 2 (tabla 4). Los valores más bajos y los 
coeficientes de variación más altos se obtuvieron para el arreglo 1, cuya resistencia promedio es sólo el $75 \%$ de la obtenida para el arreglo 2 y, dada la enorme dispersión en sus resultados, la resistencia índice $v^{*}{ }_{m}$ conseguida para el arreglo 1 es aproximadamente la mitad de la obtenida para el arreglo 2.

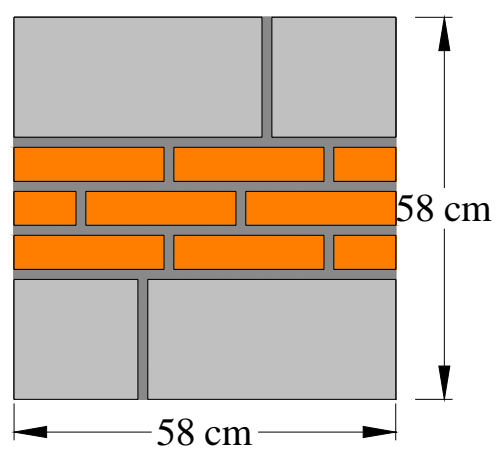

a) Murete con arreglo 1

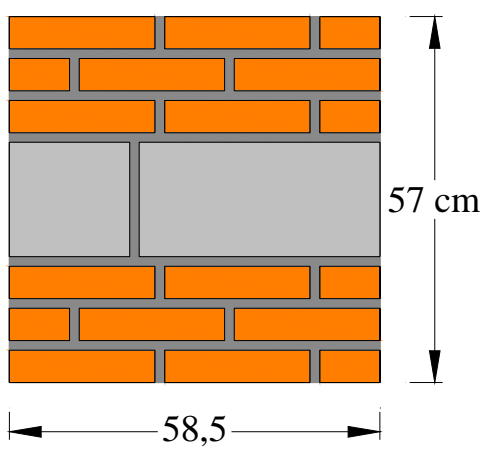

b) Murete con arreglo 2

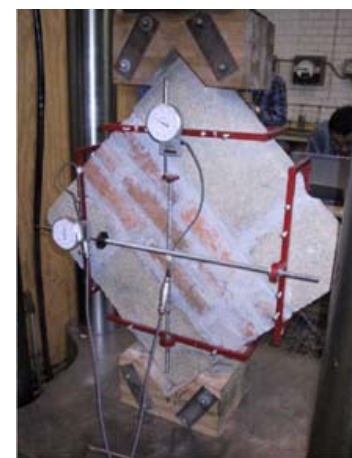

c) murete con arreglo 1 listo para el ensaye

Figura 10. Dimensiones y configuración de los muretes para el ensaye a compresión diagonal

Tabla 4. Resistencia de muretes a tensión diagonal

\begin{tabular}{cccc}
\hline Arreglo & $\begin{array}{c}\text { Resistencia media } \\
v_{\mathrm{m}}\left(\mathrm{kg} / \mathrm{cm}^{2}\right)\end{array}$ & $C_{V}$ & $\begin{array}{c}v^{*}{ }^{*} \\
\mathrm{~kg} / \mathrm{cm}^{2}\end{array}$ \\
\hline 1 & 2.9 & 0.51 & 1.28 \\
2 & 3.86 & 0.18 & 2.57 \\
$1 \mathrm{y} 2$ & 3.35 & 0.37 & 1.74 \\
\hline
\end{tabular}

Tabla 5. Módulo de cortante

\begin{tabular}{ccc}
\hline Arreglo & $\begin{array}{c}G_{m} \text { promedio } \\
\mathrm{kg} / \mathrm{cm}^{2}\end{array}$ & $C_{v}$ \\
\hline 1 & $4,283.8$ & 0.45 \\
2 & $4,006.7$ & 0.19 \\
$1 \mathrm{y} 2$ & $4,227.3$ & 0.34 \\
\hline
\end{tabular}

La diferencia en los resultados reportados se debe a que el modo de falla dominante observado entre ambos arreglos fue diferente. Para los muretes con el arreglo 1, el modo de falla que predominó fue por deslizamiento de la mampostería (figuras 11a y 11b), debido a una débil adherencia entre el mortero y las piezas de mampostería, mientras que en los muretes del arreglo 2 el modo de falla predominante fue por tensión diagonal (figura 11c); solamente en uno de los nueve muretes se presentó la falla por esfuerzos tangenciales en las juntas.

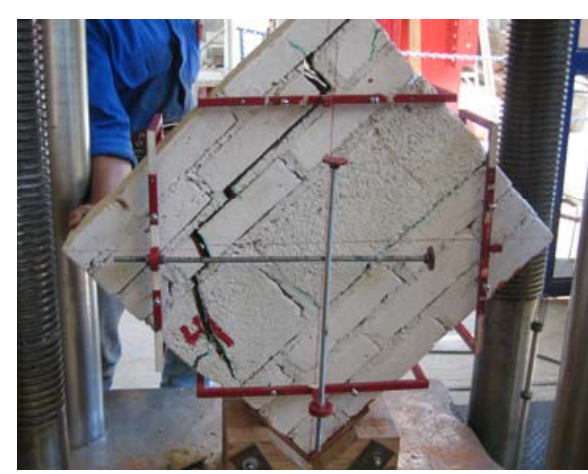

a) Arreglo 1, falla por deslizamiento

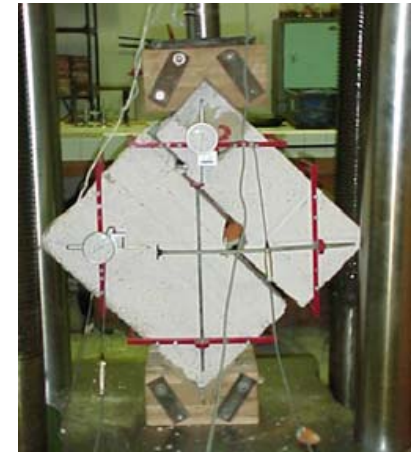

b) Arreglo 1, falla por deslizamiento

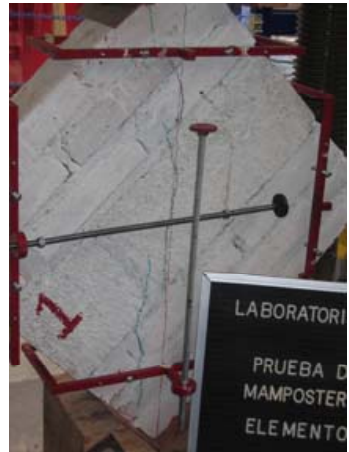

c) Arreglo 2, falla por tensión diagonal

Figura 11. Fallas observadas en los muretes durante los ensayes a compresión diagonal 
En virtud de lo anterior, queda la duda razonable de qué tan representativo es este ensaye para determinar la resistencia índice a cortante de esta modalidad de mampostería, lo cual sólo se puede contestar a partir de confrontar estos resultados con los de los ensayes de los muros ante carga lateral cíclica, lo que se presenta secciones más adelante.

\section{DISEÑO Y CONSTRUCCIÓN DE MUROS DE PRUEBA}

De acuerdo con el Apéndice A de las NTCM-2004, en el que se especifica el ensaye de al menos un espécimen para cada configuración característica del refuerzo o condiciones de borde, se fabricaron dos muros de mampostería confinada y combinada de $2.30 \mathrm{~m}$ de longitud por $2.30 \mathrm{~m}$ de altura, conforme a los arreglos que se muestran en la figura 12. La altura y el ancho de los muros de prueba estuvieron limitados por las características del dispositivo de carga. La nomenclatura asignada a cada espécimen se hizo de acuerdo con el arreglo de la mampostería (Mampostería Combinada y Confinada, MCC), orden de ensaye (1 o 2) y a las características de cada espécimen.

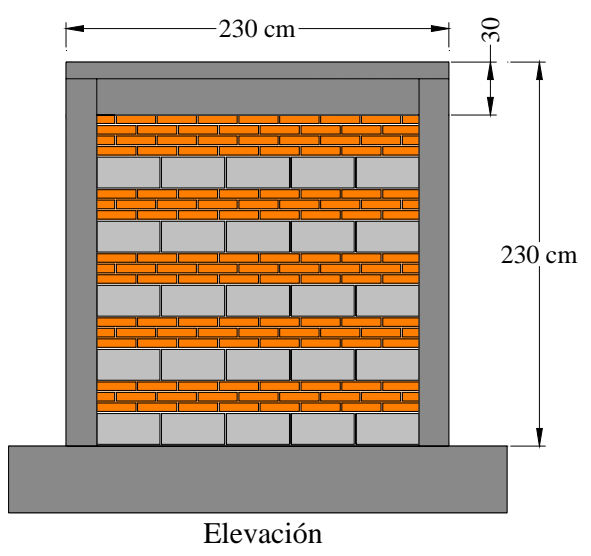

a) Arreglo del muro MCC-1

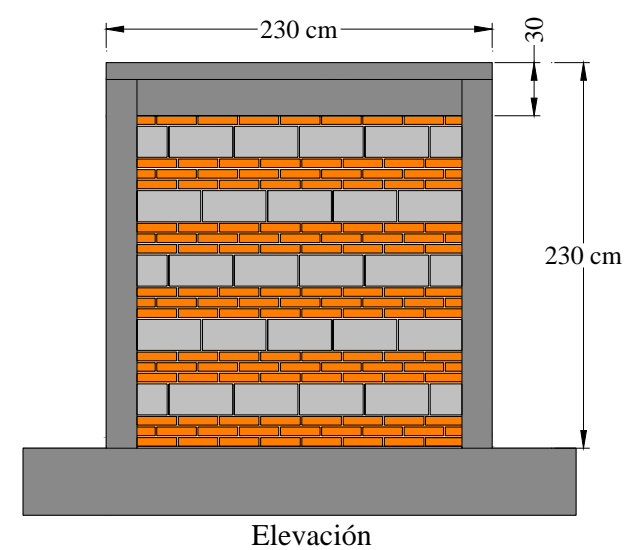

b) Arreglo del muro MCC-2

Figura 12. Arreglos de los muros de mampostería combinada ensayados

Se construyeron los especimenes de manera que sus características generales coincidieran lo más posible con las de muros de tabique rojo confinados ensayados previamente en el Cenapred (modelo M-0E6, Flores y Alcocer 2001), con la finalidad de poder hacer comparaciones entre estas distintas modalidades. De hecho, las dimensiones generales y características de los elementos confinantes y dala de cimentación fueron tomadas de informes publicados en dicha institución (Flores y Alcocer 2001), pero se adaptaron de acuerdo con las condiciones del dispositivo de carga del Laboratorio de Estructuras de la Universidad Autónoma Metropolitana. Por tanto, los muros se apoyaron sobre una viga de reacción de concreto reforzado, diseñada y fabricada in situ (figura 13).

El confinamiento consiste de dos castillos de sección rectangular de dimensiones de 12 x $20 \mathrm{~cm}$ y un sistema de dala-losa en la parte superior. La sección de la dala fue de 12 x $20 \mathrm{~cm}$ y la losa de 80 x 10 $\mathrm{cm}$ de ancho y peralte, respectivamente. Los armados de estos elementos se resumen en las tabla 6. El refuerzo longitudinal provisto a dalas, castillos y losa consiste de cuatro varillas del \# 3 (3/8” de diámetro) como acero longitudinal y de estribos del \#2 a cada $20 \mathrm{~cm}$ para el acero transversal. La figura 14 muestra en detalle el número de varillas y la separación entre el refuerzo longitudinal y el transversal en el caso de las dalas, castillos y losa. Las cuantías calculadas con este tipo de refuerzo cumplen con los mínimos 
establecidos en la sección 3.3 de las NTCM-2004 para considerar un muro de mampostería como confinado.

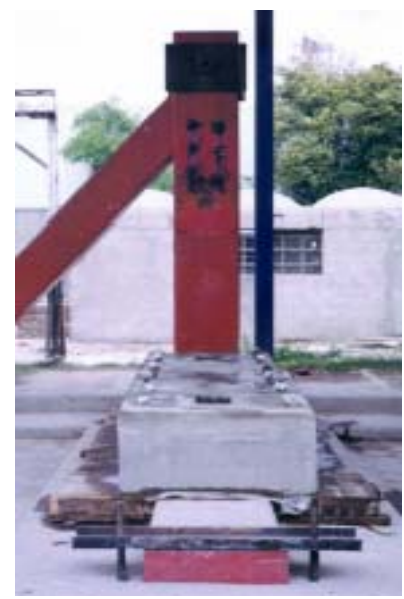

a) Viga de reacción colada en el dispositivo

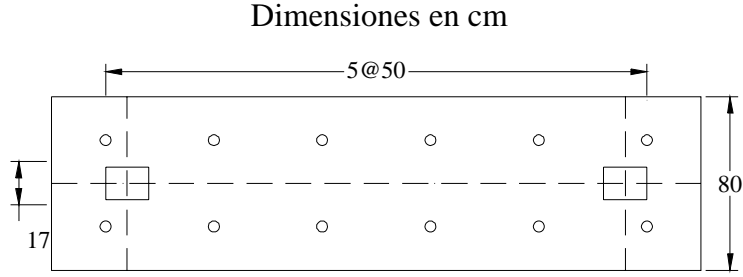

b) Planta

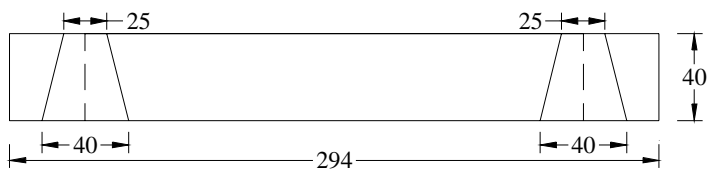

c) Elevación

Figura 13. Detalles de la viga de cimentación

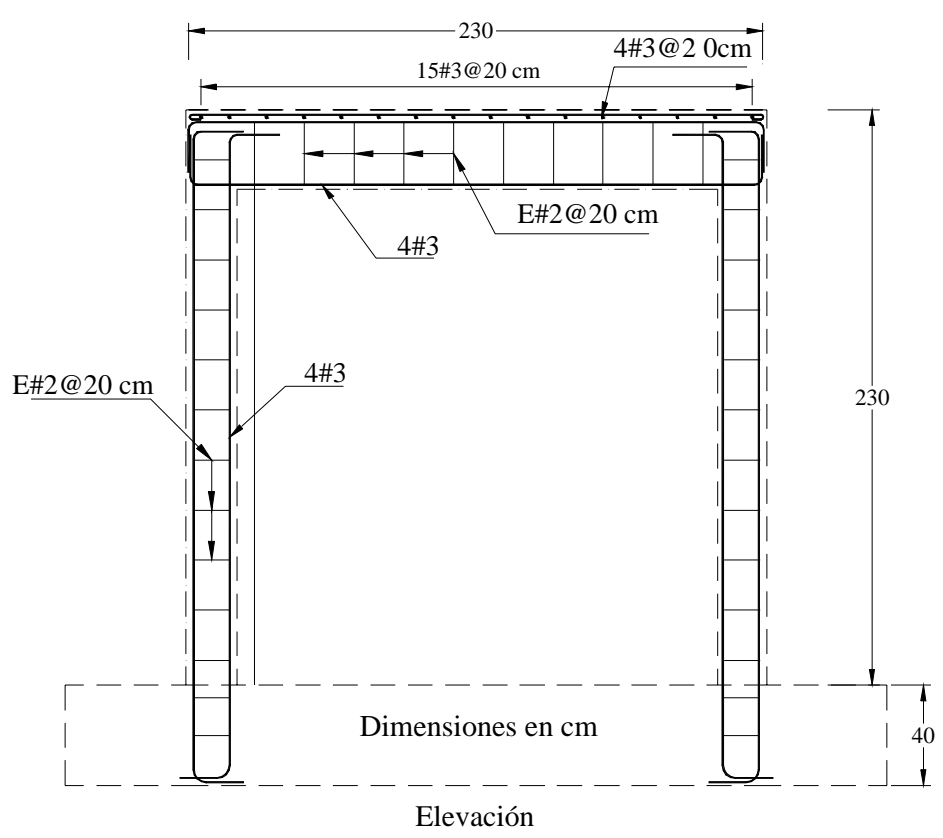

Figura 14. Armado de los elementos confinantes

El sistema dala-losa fue necesario para representar de manera más aproximada el modelo con la realidad, y transmitir las cargas laterales y verticales al muro. En el modelo MCC-1 se coló por separado la losa y la dala, colocando a lo largo de ésta, conectores de varilla del \#4 para evitar el deslizamiento al momento de la aplicación de la carga. Para el modelo MCC-2, se coló de forma monolítica el sistema dala-losa. En la figura 15 se muestran la geometría y dimensiones de los modelos de los muros, así como los detalles en planta y elevación. 
Resistencia y deformación de muros de mampostería combinada y confinada sujetos a cargas laterales

Tabla 6. Características del refuerzo en los especimenes

\begin{tabular}{|c|c|c|c|c|c|c|}
\hline \multirow[t]{2}{*}{ Muro } & \multicolumn{2}{|c|}{ Refuerzo en castillos } & \multicolumn{2}{|c|}{ Refuerzo en dalas } & \multicolumn{2}{|c|}{ Refuerzo en losas } \\
\hline & Longitudinal & Transversal & Longitudinal & Transversal & Longitudinal & Transversal \\
\hline MCC-1 & $4 \# 3$ & E\#2@ $20 \mathrm{~cm}$ & $4 \# 3$ & E\#2@20 cm & 4\#3@ 15 cm & 16\#4@15 cm \\
\hline MCC-2 & $4 \# 3$ & E\#2@ 20 cm & $4 \# 3$ & E\#2@20 cm & 4\#3@15 cm & 16\#3@15 cm \\
\hline
\end{tabular}

\#3: Acero de 3/8 de pulgada de diámetro, del grado $42\left(f_{y}=4,200 \mathrm{~kg} / \mathrm{cm}^{2}\right)$

E\#2: Alambrón de 2/8 de pulgada de diámetro, del grado $22\left(f_{y}=2,200 \mathrm{~kg} / \mathrm{cm}^{2}\right)$

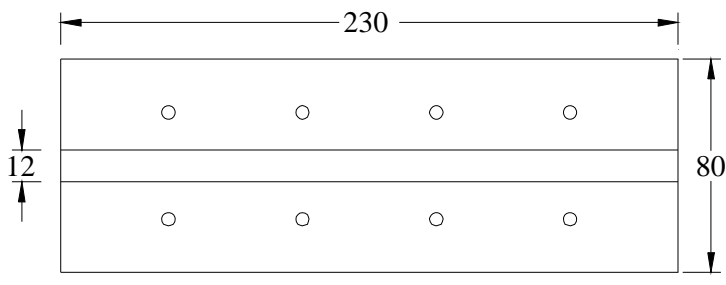

Dimensiones en cm

a) Planta

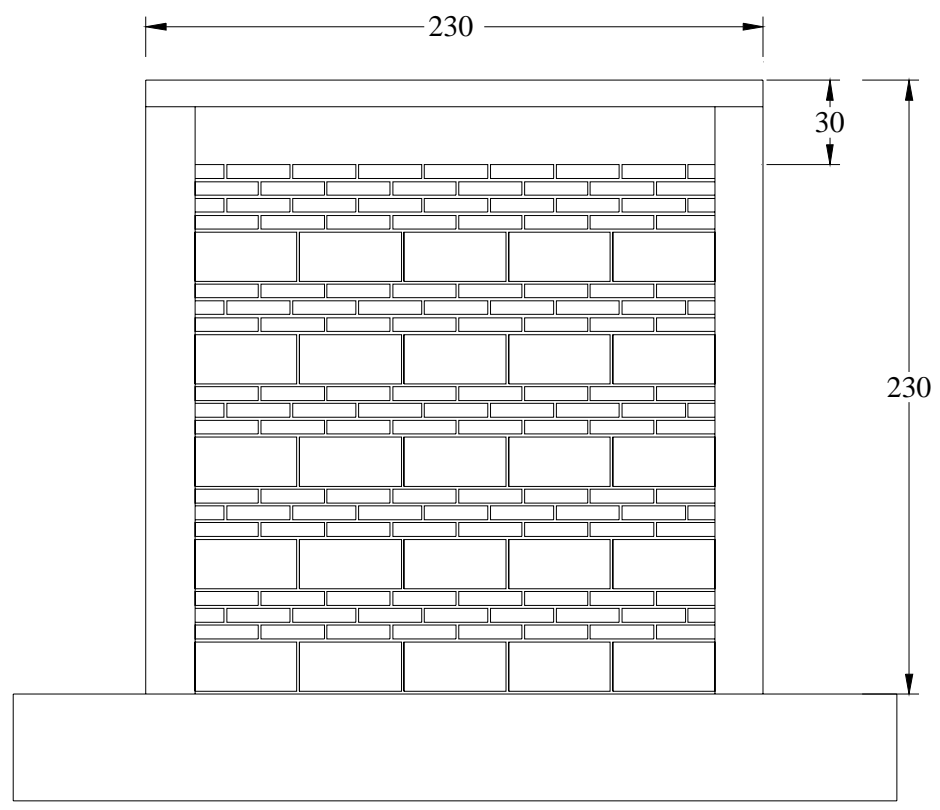

b) Elevación

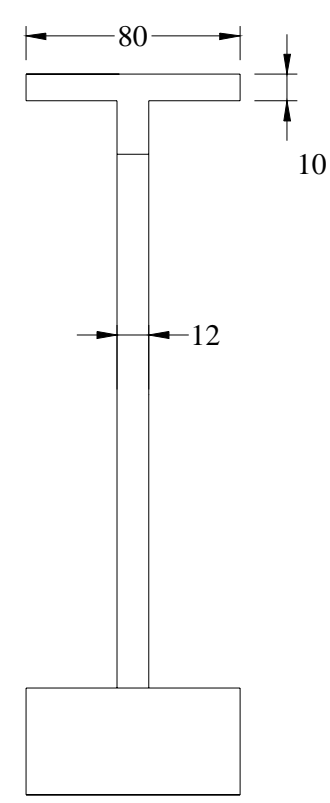

c) Corte

Figura 15. Geometría y dimensiones generales de los especimenes

Como se presentó en la sección anterior, los materiales empleados en la fabricación de los muros fueron muestreados con objeto de determinar sus propiedades mecánicas. El concreto utilizado en los elementos de confinamiento, así como en la losa y viga de reacción, se elaboró en el Laboratorio de Estructuras de la UAM y se dosificó para que alcanzaran la resistencia de diseño. En la tabla 7 se resumen las resistencias de diseño y las obtenidas de los ensayes a los cilindros, cuyos resultados se reportan en detalle en Juárez (2007). 
Arturo Tena Colunga, Artemio Juárez Ángeles y Víctor Hugo Salinas Vallejo

Tabla 7. Resistencia promedio a la compresión de cilindros de concreto

\begin{tabular}{|c|c|c|c|}
\hline \multirow[b]{2}{*}{ Elemento estructural } & \multirow{2}{*}{$\begin{array}{c}\text { Resistencia de diseño } \\
f_{c}^{\prime} \\
\mathrm{kg} / \mathrm{cm}^{2}\end{array}$} & \multicolumn{2}{|c|}{ Espécimen } \\
\hline & & $\begin{array}{c}\text { MCC-1 } \\
\text { Resistencia media } \\
\mathrm{kg} / \mathrm{cm}^{2}\end{array}$ & $\begin{array}{c}\text { MCC-2 } \\
\text { Resistencia media } \\
\mathrm{kg} / \mathrm{cm}^{2}\end{array}$ \\
\hline Viga de reacción & 300 & 340.8 & 341 \\
\hline Castillos & 150 & 182.2 & 140 \\
\hline Dalas & 250 & 239.9 & 248 \\
\hline Losa & 250 & 242.8 & 248 \\
\hline
\end{tabular}

\section{PREDICCIÓN DE RESULTADOS}

\section{Fuerza cortante resistida por la mampostería confinada}

Con el fin de contar con un estimado de la resistencia esperada para los especimenes, se empleó la expresión de las NTCM-2004 para calcular la capacidad de muros a cortante, la que está calibrada para obtener la carga de agrietamiento de muros de mampostería confinada basados en un solo tipo de pieza:

donde:

$$
V_{m R}=F_{R}\left(0.5 v_{m}^{*} A_{T}+0.3 P\right) \leq 1.5 F_{R} v_{m}^{*} A_{T}
$$

$F_{R} \quad$ factor de reducción de resistencia; se tomó igual a 1.0 para la predicción en estos ensayes;

$P \quad \quad$ carga vertical de compresión sobre el muro;

$v_{m}{ }^{*} \quad$ resistencia a compresión diagonal de la mampostería;

$A_{T} \quad$ área transversal del muro (área en planta = longitud por espesor del muro)

Tomando los valores mínimos y máximos de $v^{*}{ }_{m}$ reportados en la tabla 4 se realizaron los cálculos correspondientes para obtener las resistencias esperadas a cortante de la mampostería combinada de cada uno de los arreglos de los muretes ensayados. La carga vertical de compresión aplicada a los muros fue de 2.2 ton aproximadamente, para un esfuerzo de compresión promedio de $0.8 \mathrm{~kg} / \mathrm{cm}^{2}$. En la tabla $8 \mathrm{se}$ resume la resistencia lateral nominal esperada para los especimenes, calculadas conforme a la expresión 1.

Tabla 8. Resistencias nominales esperadas para los muros de prueba

\begin{tabular}{|c|c|c|c|c|c|}
\hline & $\begin{array}{c}A_{T} \\
\mathrm{~cm}^{2}\end{array}$ & $\begin{array}{c}P \\
\text { ton }\end{array}$ & $F_{R}$ & $\begin{array}{c}v_{m}^{*} \\
\mathrm{~kg} / \mathrm{cm}^{2}\end{array}$ & $\begin{array}{l}\mathrm{V}_{\mathrm{mR}} \\
\text { ton }\end{array}$ \\
\hline Mínima, muros MCC-1 y MCC-2 & 2,760 & 2.23 & 1 & 1.28 & 2.44 \\
\hline Máxima, muros MCC-1 y MCC-2 & 2,760 & 2.23 & 1 & 2.57 & 4.22 \\
\hline
\end{tabular}

\section{Resistencia a flexocompresión en el plano del muro}

Considerando la posibilidad de una falla a flexocompresión, se estimó el momento nominal conforme al método optativo de las NTCM-2004 para definir un diagrama de interacción simplificado, indicado en la sección 5.3.2.2 de dichas normas, ya que los muros contaban con el acero longitudinal de 
los castillos colocado simétricamente en los extremos. Las ecuaciones establecidas en las NTCM-2004 para este método aproximado son:

$$
\begin{array}{lll}
M_{R}=F_{R} M_{0}+0.3 P_{u} ; & \text { si } & 0 \leq P_{u} \leq \frac{P_{R}}{3} \\
M_{R}=\left(1.5 F_{R} A_{s} f_{y} d^{\prime}+0.15 P_{R} d\right)\left[1-\frac{P_{u}}{P_{R}}\right] ; & \text { si } & P_{u} \geq \frac{P_{R}}{3} \\
M_{0}=A_{S} f_{y} d^{\prime} & & \\
P_{R}=F_{R} F_{E}\left(f_{m}^{*} A_{T}+\sum A_{s} f_{y}\right) & &
\end{array}
$$

En los cálculos se consideró que $F_{R}=1$ y $F_{E}=0.6$ (muro exterior). De acuerdo con las propiedades de la mampostería y el nivel de carga axial aplicado, rigió la ecuación 2, los resultados se reportan en la tabla 9 .

Tabla 9. Resistencias nominales esperadas a flexocompresión para los muros de prueba

\begin{tabular}{cccccccccccc}
\hline Muros & $\begin{array}{c}A_{T} \\
\mathrm{~m}^{2}\end{array}$ & $\begin{array}{c}P_{u} \\
\text { ton }\end{array}$ & $F_{R}$ & $F_{E}$ & $\begin{array}{c}\mathrm{f}_{\mathrm{m}}^{*}{ }^{2} \\
\mathrm{~cm}^{2}\end{array}$ & $\begin{array}{c}f_{y} \\
\mathrm{~kg} / \mathrm{cm}^{2}\end{array}$ & $\begin{array}{c}A_{s} \\
\mathrm{~cm}^{2}\end{array}$ & $\begin{array}{c}M_{0} \\
\text { ton-m }\end{array}$ & $\begin{array}{c}M_{R} \\
\text { ton-m }\end{array}$ & $\begin{array}{c}H \\
\mathrm{~m}\end{array}$ & $V^{2}$ \\
\hline $\begin{array}{l}\text { MCC-1 } \\
\text { MCC-2 }\end{array}$ & 0.28 & 2.23 & 1 & 0.6 & 19.7 & 4200 & 1.03 & 9.12 & 10.6 & 2.3 & 4.6 \\
\hline
\end{tabular}

${ }^{1}$ media de la resistencia a compresión de la mampostería combinada, correspondiente a los dos tipos de arreglo ensayados (tabla 2)

${ }^{2}$ cortante asociado al momento nominal resistente por flexocompresión $\left(V=M_{R} / H\right)$

El cortante teórico asociado a una falla por flexocompresión (tabla 9) es mayor que el teórico obtenido para una falla por cortante (tabla 8), por lo que se debía esperar que en los ensayes el modo dominante de falla fuera por cortante, como efectivamente sucedió, como se ilustra y discute más adelante.

\section{INSTRUMENTACIÓN}

La instrumentación externa de los muros estaba constituida por nueve transductores de desplazamiento (LVDT) de tipo carátula y de una celda de carga. Los transductores de desplazamiento se colocaron con el fin de medir deformaciones de flexión y distorsiones angulares, como se ilustra en la figura 16. La deformación angular de los tableros, se calculó a partir de las lecturas de los transductores de desplazamiento ubicados sobre las diagonales de los muros (T5 y T6). La rotación local en la base de los castillos se registró con dos transductores ubicados en los extremos inferiores de los castillos (T1 y T3). También se colocaron micrómetros para medir el deslizamiento del muro en su base (T4) y el posible deslizamiento de la viga de cimentación (T8), para medir la distorsión (T2 y T7) así como el desplome durante la prueba (T9).

Dentro de la instrumentación externa, se colocó una celda de carga para el segundo muro MCC-2, con la que se logró tomar lecturas electrónicamente y tener una correspondencia de datos más precisa. En la figura 18 se muestra la ubicación de esta celda de carga.

La instrumentación interna consistió de deformímetros eléctricos (strain gages) adheridos a las barras de refuerzo longitudinal de castillos y dala. En la figura 17 se muestra la ubicación de los 12 
deformímetros eléctricos colocados en el refuerzo longitudinal de los elementos confinantes de los muros de prueba. Al igual que los transductores externos, estos instrumentos de medición se conectaron al dispositivo de adquisición de datos TDS.

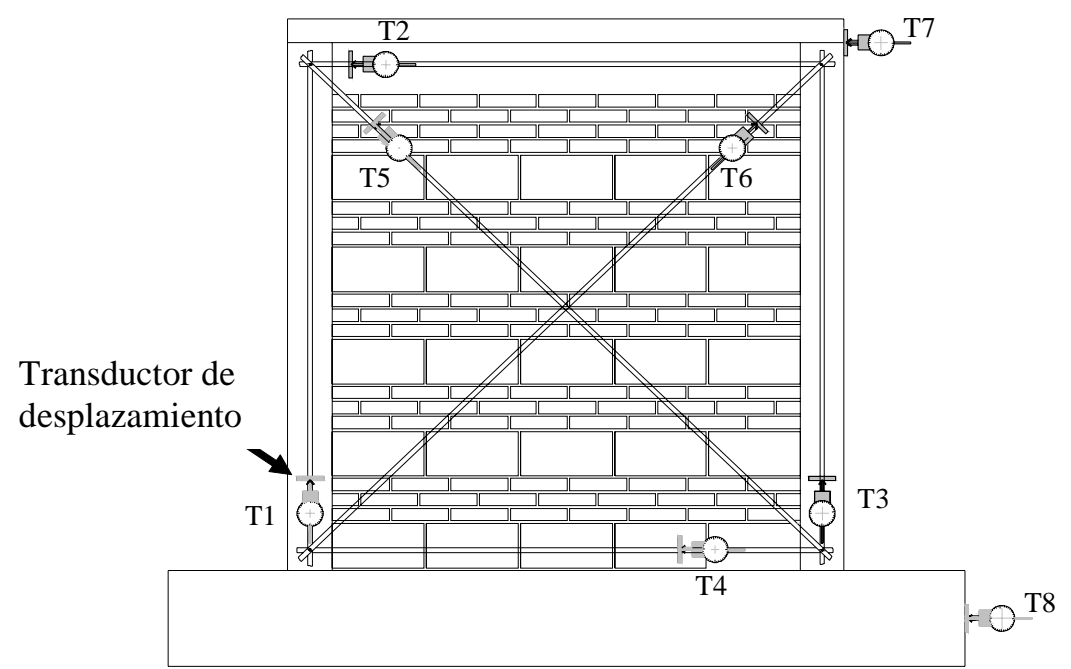

Figura 16. Instrumentación externa usada en ambos muros

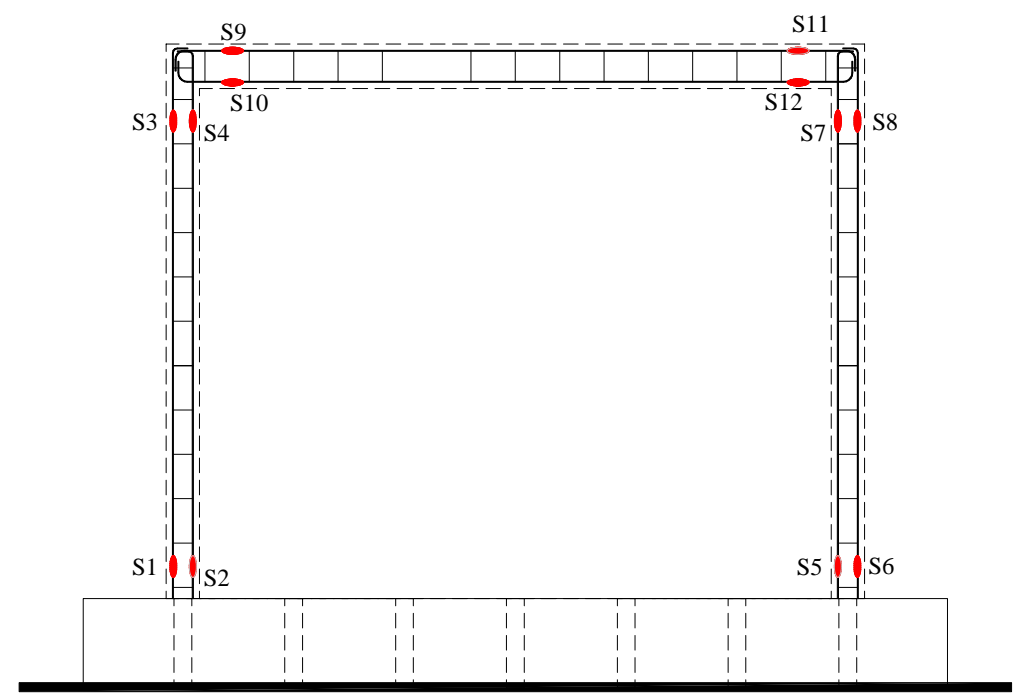

Figura 17. Instrumentación interna (ubicación de deformímetros eléctricos)

\section{ENSAYE ANTE CARGA LATERAL CÍCLICA}

El ensaye de los muros se llevó a cabo en el Laboratorio de Estructuras de la Universidad Autónoma Metropolitana Azcapotzalco utilizando el dispositivo ilustrado en la figura 18. Cabe señalar que son los dos primeros muros ensayados en la Universidad Autónoma Metropolitana en condiciones 
semejantes a los estudiados en el Cenapred y los primeros muros de mampostería combinada ensayados en México y en el mundo.

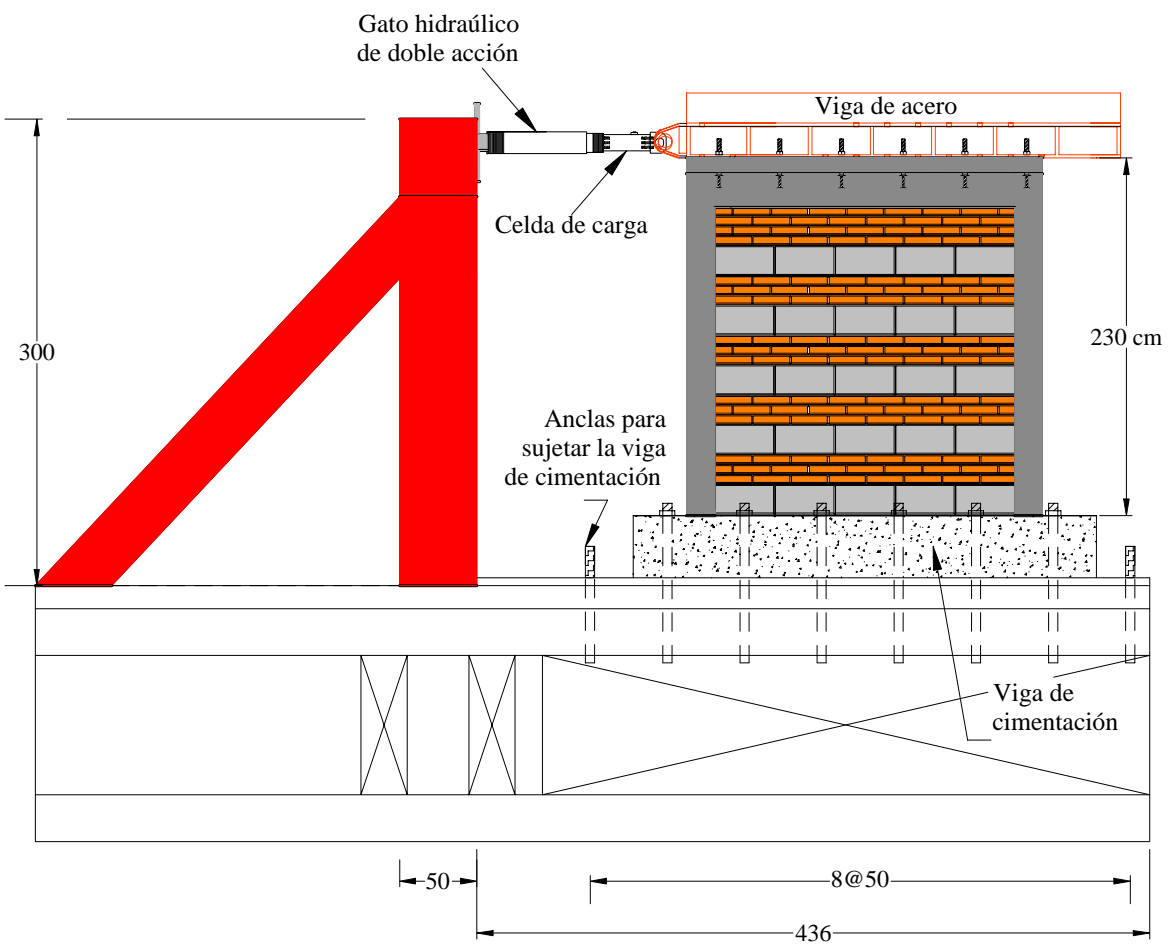

Figura 18. Dispositivo de carga, vista en elevación

Para la aplicación de la carga vertical de compresión, así como la lateral, se contó con una viga de acero anclada en la losa (figura 18). Para la aplicación de la carga horizontal se utilizó un gato hidráulico de doble acción de 20 ton, apoyado en la columna vertical del dispositivo de carga, como se muestra en la figura 18. El gato se controló con un sistema hidráulico, que aplicó la carga contra una viga de acero sujeta a lo largo de la dala-losa del muro; con ello, se proporcionó una carga horizontal distribuida a lo largo de la parte superior del muro. Asimismo, para ambos muros de prueba se aplicó carga vertical uniformemente repartida mediante el peso propio de la viga de acero y el peso de lingotes de acero que se colocaron sobre esta viga de carga. Las cargas verticales aplicadas fueron 2.2 ton para el primer muro (MCC-1) y 2.0 ton para el segundo (MCC-2).

Los ensayes fueron cíclicos reversibles con una repetición en cada incremento, en congruencia con lo especificado en la secuencia de cargas que recomiendan las NTCM-2004 en su Apéndice A para la evaluación de sistemas con base en muros de mampostería, donde los primeros ciclos se controlaron por carga hasta el primer agrietamiento, para después controlar por deformación hasta la falla.

De acuerdo con la predicción de la carga de agrietamiento, para el muro MCC-1 los dos primeros ciclos se aplicaron a una carga del $25 \%$ de la que se predijo al agrietamiento, los dos siguientes, al 50\%, y así sucesivamente hasta alcanzar el primer agrietamiento, el cual ocurrió a una carga de 5.07 ton, la que excedió un 20 \% la máxima estimada (tabla 8). Posteriormente, el ensaye se controló por distorsión, iniciando con 0.002, con incrementos de 0.001 hasta la falla. En la figura 19 se observa que en el control por carga se aplicaron cinco ciclos, cada uno con repetición. Para cada distorsión se aplicaron también dos ciclos, hasta alcanzar una distorsión de 0.006, que fue la de falla. 
Para el muro MCC-2 se siguió el mismo patrón de carga hasta llegar a la carga de agrietamiento, la cual fue de 5 ton. Para las distorsiones, el patrón empleado se presenta en la figura 20. Como se observa, sólo se alcanzó la distorsión de 0.006 en el empuje de los gatos, ya que el sistema hidráulico, presentó problemas y no se logró llegar mas allá de una distorsión 0.004 para cuando el gato jala al muro (coloquialmente definido como "jale").

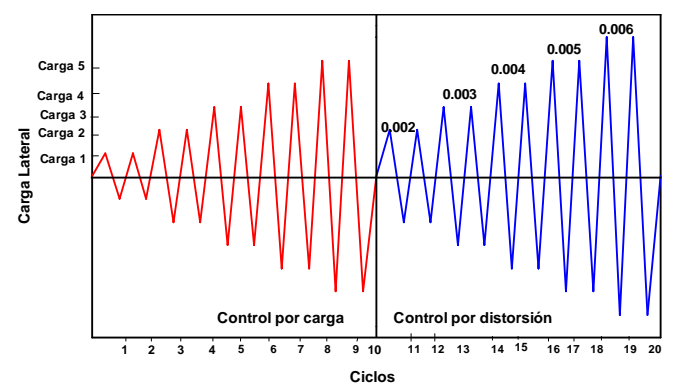

Figura 19. Historia de cargas y distorsiones, muro MCC-1

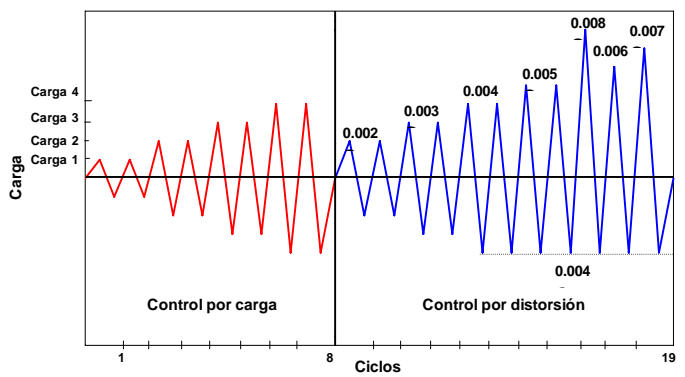

Figura 20. Historia de cargas y distorsiones, muro MCC-2

\section{Comportamiento histerético}

El comportamiento histerético observado en el muro MCC-1 (figura 21a) presenta lazos de forma casi simétrica hasta alcanzar una distorsión de $0.6 \%$. Al iniciar la aplicación del segundo ciclo de distorsión de $0.6 \%$, el muro presentó en el primer semiciclo un deslizamiento importante en su altura media, de donde la grieta diagonal se extendió súbitamente hacia los extremos superior e inferior de los castillos que ya habían sido penetrados en ciclos anteriores, obteniéndose de esta manera el estado último de falla. La penetración más crítica para la estabilidad del muro se presentó en el extremo superior de uno de los castillos (figura 22a). Los datos capturados en estas condiciones (medio ciclo) son los que se presentan con línea punteada en la figura 21a. Dado que el muro ya había fallado, no se logró completar el segundo ciclo a esta deformación.

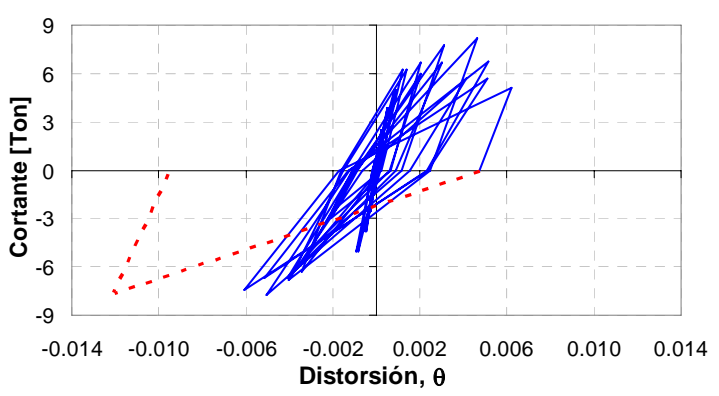

a) Muro MCC-1

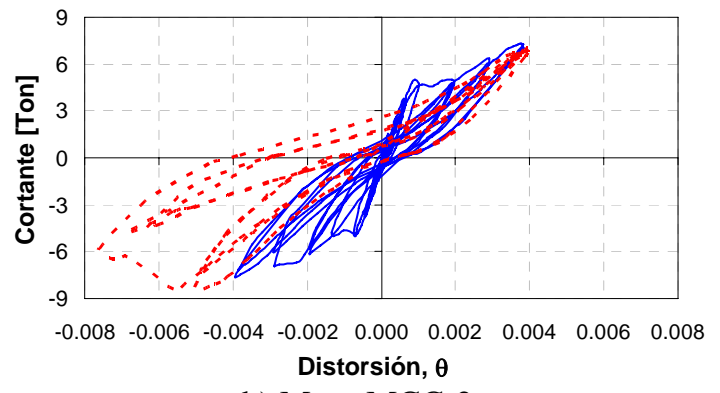

b) Muro MCC-2

Figura 21. Lazos de histéresis de los dos muros

El muro MCC-2 presentó una buena simetría en los ciclos histeréticos (“empuje” y "jale”) hasta alcanzar una distorsión de 0.004 (figura 21b). Al realizar la repetición para la distorsión de $0.5 \%$, el sistema no logró desarrollar esta distorsión al momento de jalar el espécimen. La prueba se continuó hasta alcanzar la distorsión de $0.8 \%$ en los ciclos de empuje. Estos ciclos asimétricos se muestran con línea punteada en la figura $21 \mathrm{~b}$. 
Cabe señalar que la menor resolución de los lazos de histéresis presentados para el muro MCC-1 (figura 21a) con respecto a los del muro MCC-2 (figura 21b) se deben a que en el ensaye del muro MCC-1 no fue posible colocar una celda de carga, por lo que las cargas aplicadas, se obtuvieron a partir de las lecturas de un manómetro previamente calibrado, las cuales se tomaron al instante que se alcanzó una distorsión objetivo. Como se mencionó previamente, para el ensaye del muro MCC-2 fue posible colocar una celda de carga (figura 18), con la que se logró tomar todas las lecturas electrónicamente y capturar todos los datos de manera más precisa.

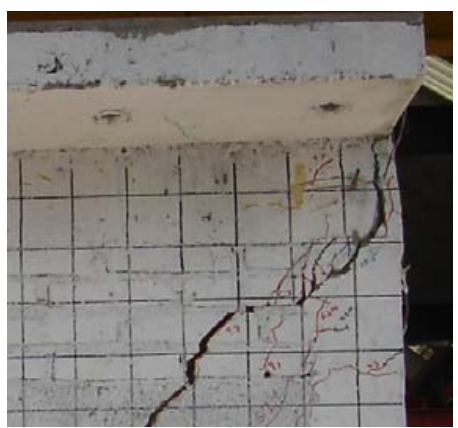

a) Grieta en castillo, extremo superior

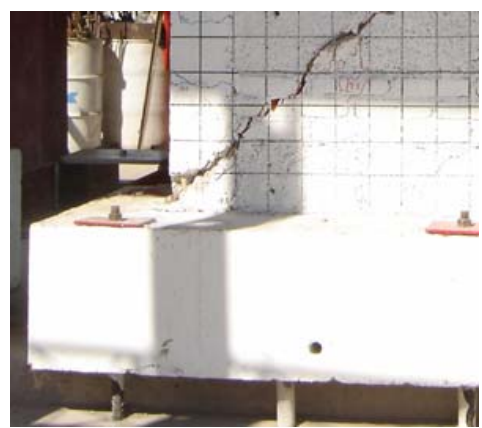

b) Grieta en castillo, extremo inferior

Figura 22. Estado último de falla en los castillos del muro MCC-1

\section{Envolvente de la curva carga lateral-distorsión}

En el primer muro ensayado, la etapa elástica llegó hasta una distorsión de $0.09 \%$ correspondiente a una carga de 5.0 ton (figura 23a). El primer gran agrietamiento ocurrió a una carga de 6.3 ton y una distorsión de $0.34 \%$; el espesor de esta primera gran grieta fue de $5 \mathrm{~mm}$. En la etapa inelástica, que correspondió al control por distorsión, el espesor de grieta que se comenzó a registrar fue de $1.5 \mathrm{~mm}$ en el bloque y $2.5 \mathrm{~mm}$ en el tabique, hasta llegar a $22 \mathrm{~mm}$ en ambas piezas. En la figura 23a se presentan las cargas máximas en ambos cuadrantes; en el caso de empuje, se aprecia el deslizamiento que ocurrió al final del ensaye por la penetración del castillo, el cual inició a una distorsión de $0.6 \%$ y la lectura final cuando se retiró de inmediato la aplicación de la carga llegó a ser de 1.2\%, en condiciones de claro colapso, motivo por el cual se dio por terminado el ensaye. Las cargas máximas correspondientes a empuje y jale del espécimen MCC- 1 fueron de 7.7 ton $(\theta=0.005)$ y 8.2 ton $(\theta=0.0046)$, respectivamente.

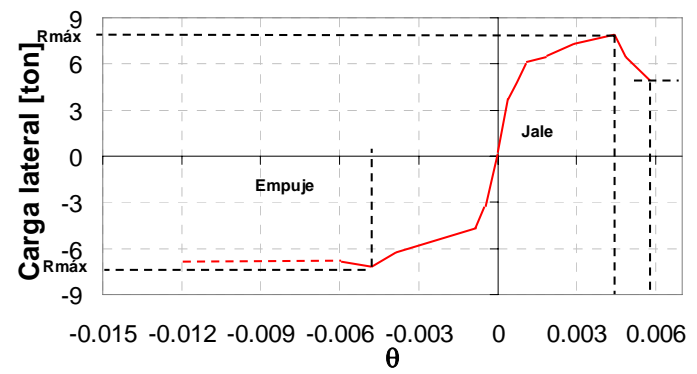

a) Muro MCC-1

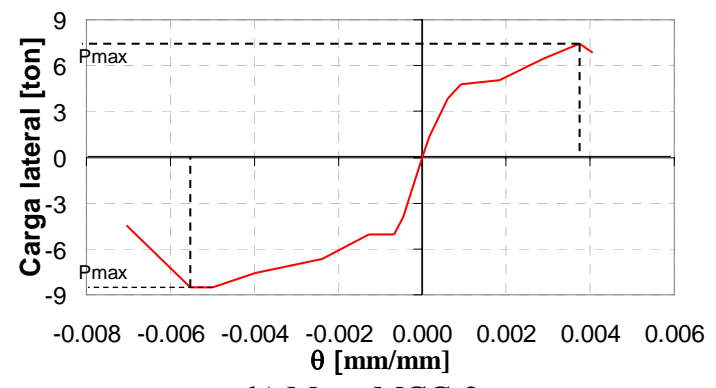

b) Muro MCC-2

Figura 23. Envolventes carga-deformación de los muros MCC-1 y MCC-2 
Para el muro MCC-2 (figura 23b), la etapa elástica llegó hasta una distorsión de $0.07 \%$ y una carga de 5.0 ton. El primer gran agrietamiento ocurrió bajo una carga de 5.0 ton y una distorsión de $0.09 \%$ (al momento de jalar el espécimen); el espesor de esta grieta fue de $1.5 \mathrm{~mm}$ en el bloque y $2.5 \mathrm{~mm}$ en el tabique. A partir de esta etapa el control se continuó por distorsión, hasta llegar a la carga máxima de 8.35 ton con distorsión de $0.6 \%$ y un ancho de grieta de $15 \mathrm{~mm}$ en el tabique y $10 \mathrm{~mm}$ en el bloque. Las cargas máximas correspondientes a empuje y jale del espécimen MCC- 1 fueron de 7.3 ton $(\theta=0.0038)$ y 8.4 ton $(\theta=0.0056)$, respectivamente.

\section{Degradación de rigidez de ciclo}

Un parámetro importante en la evaluación de estructuras existentes, como en el diseño de nuevas estructuras de mampostería, es la degradación de rigidez, debido a que el daño de las mismas está asociado a su capacidad de absorber cargas laterales a través de su deformación. A menudo, se toman valores de distorsión permisibles en lo reglamentos que limitan el desplazamiento lateral de las edificaciones para evitar un daño severo en la mampostería. Sin embargo, es necesario realizar un análisis a fondo para determinar los valores que deben permitirse a dichas estructuras. En este apartado se muestra la degradación de rigidez en función del nivel de distorsión medido para los dos muros de mampostería combinada y confinada ensayados.

La rigidez de ciclo se calcula como la pendiente de la secante que une los puntos de máxima distorsión, en sentidos positivo y negativo para un mismo ciclo. En la figura 24a se presenta la curva de degradación de rigidez para los primeros ciclos y para las repeticiones del muro MCC-1. Se observa que aún para distorsiones de 0.001 , cercanas a la aparición del primer agrietamiento, la pérdida de rigidez es importante llegando a ser aproximadamente solamente un $40 \%$ de la rigidez inicial. Para la distorsión donde inicia la penetración de grietas en castillos (0.003), la rigidez es tan sólo $20 \%$ la rigidez inicial. Cuando se desconchan los bloques (distorsión de 0.004), la rigidez efectiva puede llegar a ser de tan sólo $10 \%$ la rigidez inicial.

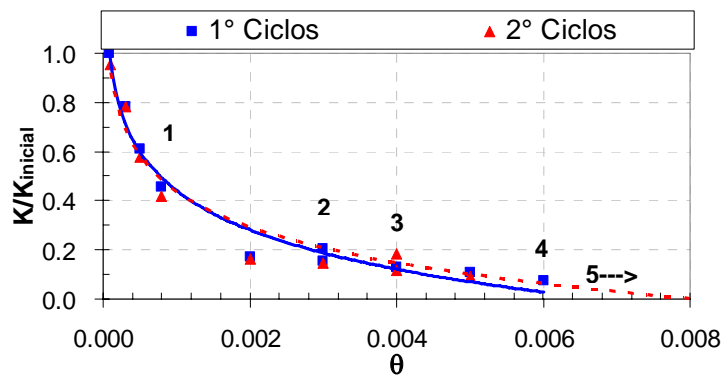

a) Muro MCC-1

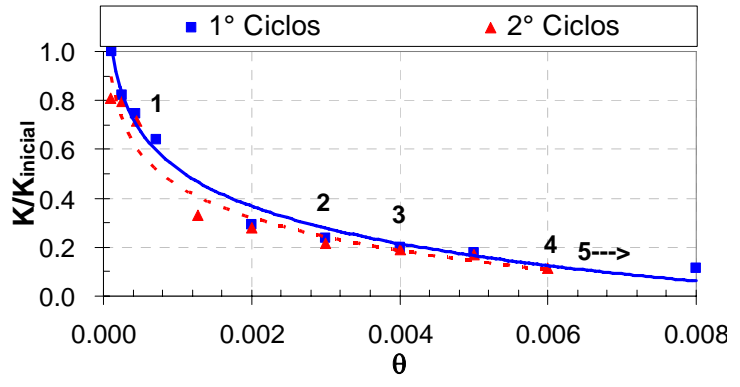

b) Muro MCC-2

1. Primera grieta diagonal

2. Inicio de la penetración de grietas en los castillos

3. Desconchamiento en los bloques

4. Penetración total de grietas en los castillos

5. Muro al borde del colapso

Figura 24. Curvas de degradación de rigidez observada para los muros MCC-1 y MCC-2

Para el muro MCC-2 (figura 24b), para la distorsión de 0.001, cercana a la aparición del primer agrietamiento, la rigidez efectiva es un poco mayor del $50 \%$ de la rigidez inicial. La distorsión donde inicia la penetración de grietas en castillos es también 0.003, aunque la rigidez efectiva en este caso es 
cercana al 25\% la rigidez inicial. Cuando se desconchan los bloques, también a una distorsión de 0.004, la rigidez efectiva en este muro fue de aproximadamente $20 \%$ la rigidez inicial.

\section{Disipación de energía}

La energía disipada por la deformación de la estructura es un parámetro muy importante, ya que a través de este concepto, se determina tanto la energía de deformación elástica recuperable, como la capacidad de energía disipada por deformación de la estructura que no es recuperable y que está ligada al daño estructural. En Juárez (2007) se presenta con detalle cómo se calcularon estos parámetros desde el punto de vista energético y cuyas bases se encuentran disponibles en libros clásicos de mecánica de sólidos (por ejemplo, Hibbeler 1998) y de dinámica estructural (por ejemplo, Chopra 1995). Entre los parámetros calculados se encuentran: a) energía de deformación elástica, b) energía de fractura, c) energía disipada equivalente (según definición del Apéndice A de las NTCM-2004), d) energía histerética acumulada y, e) amortiguamiento viscoso equivalente. En la tabla 10 se presentan los valores calculados para los primeros tres parámetros, donde se observa que ambos muros desarrollan una energía disipada equivalente mayor al valor mínimo de 0.15 establecido en el Apéndice A de las NTCM-2004 para aceptar a un sistema de mampostería como apto para resistir sismos.

Tabla 10. Disipación de energía de los muros MCC-1 y MCC-2

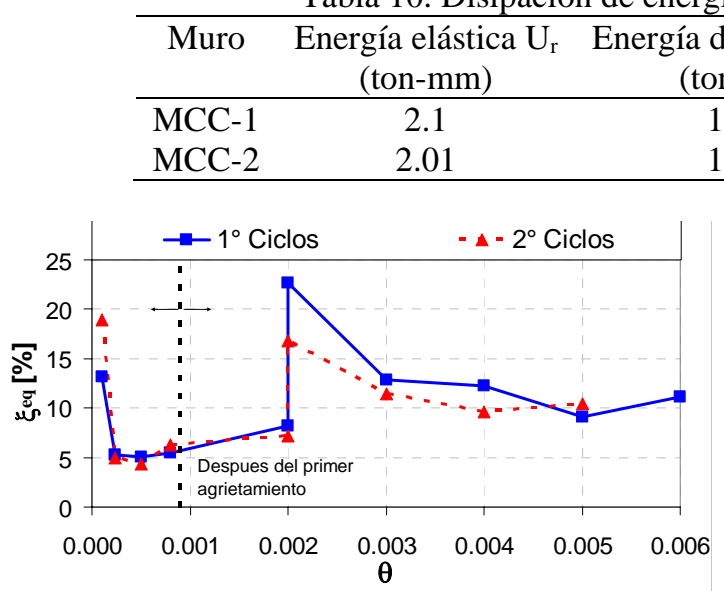

a) Muro MCC-1 gía de fractura $U_{t}$ Energía disipada equivalente ton-mm)

$\begin{array}{ll}177.5 & 0.189 \\ 109.4 & 0.314\end{array}$

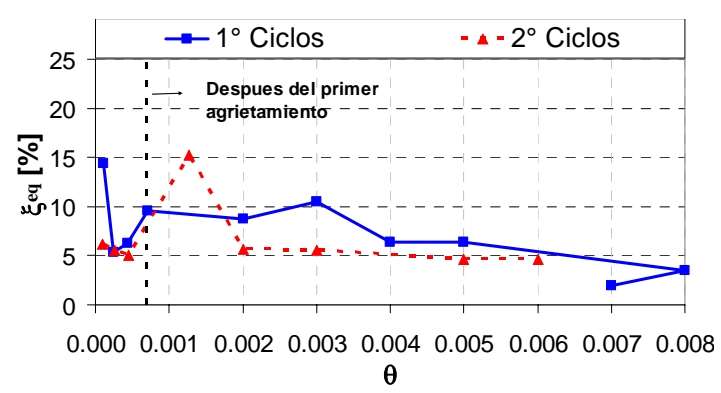

b) Muro MCC-2

Figura 25. Variación del amortiguamiento viscoso equivalente con la distorsión lateral

En la figura 25 se presenta la variación del amortiguamiento viscoso equivalente con respecto a la distorsión lateral para cada muro. El amortiguamiento viscoso equivalente se calculó a partir de la energía disipada por histéresis para cada ciclo de distorsión y distinguiendo el primer ciclo a ese nivel de distorsión con línea continua y cuadros con respecto al segundo ciclo, donde se usa línea punteada y triángulos. En general, se observa que el coeficiente de amortiguamiento viscoso equivalente depende del nivel de distorsión y que normalmente es mayor en los primeros ciclos que en los segundos, como consecuencia directa que se disipó más energía en los primeros ciclos que en los segundos (no mostrado, Juárez 2007). Se observa que para distorsiones muy bajas, el cálculo de este parámetro es algo impreciso y generalmente da valores muy altos, por lo que tomando como más representativos los valores previos al inicio del primer agrietamiento para el comportamiento elástico, el amortiguamiento viscoso equivalente elástico oscila entre $6 \%$ y $7 \%$. Para distorsiones entre $0.2 \%$ y $0.4 \%$, el valor de $\xi_{\text {eq }}$ oscila en general entre el $10 \%$ y $15 \%$ para el muro MCC-1 y entre $7 \%$ y $10 \%$ para el muro MCC-2. Se obtuvo un valor extraordinario de 23\% para el primer ciclo a una distorsión de $0.2 \%$ para el muro MCC-1, como 
consecuencia del inicio de la penetración de grietas en los castillos. Para el muro MCC-2 el amortiguamiento equivalente máximo después del primer agrietamiento fue de $15 \%$ para el segundo ciclo a una distorsión de $0.13 \%$.

\section{Índice de daño de Kwok y Ang}

La resistencia lateral de un muro de mampostería está íntimamente relacionada con su capacidad friccional. Toda vez que las grietas se extienden y se abren más, el muro ha disipado una gran cantidad de energía, fenómeno que eventualmente liderará al muro a un colapso inminente, incluso bajo fuerzas menores actuando fuera de su plano.

En este apartado, se calibra el índice de daño propuesto por Kwok y Ang (1987), que relaciona la respuesta de muros de mampostería con el nivel esperado de daño. De acuerdo con el comportamiento de la mampostería, Kwok y Ang establecen que el daño por sismo se puede expresar en función de la energía disipada y el daño causado por una excesiva deformación y lo expresan en términos de un índice de daño, definido como:

$$
\begin{aligned}
& D=D_{u}+D_{e} \\
& D_{u}=\frac{u_{m}}{u_{f}} \\
& D_{e}=\varepsilon \frac{\int d E}{q_{u} u_{f}}
\end{aligned}
$$

donde $u_{\mathrm{m}}$ es la deformación a la carga máxima, $\mathrm{u}_{\mathrm{f}}$ la deformación a la falla, $\int d E$ la energía histerética acumulada, $q_{u}$ la capacidad o resistencia última a cortante y $\varepsilon$ una constante obtenida a partir de regresión de datos experimentales. El índice de daño calculado para cada espécimen ensayado es una medida de su capacidad para resistir daño, cuando se les sujeta a cargas cíclicas reversibles (Kwok y Ang 1987).

Este índice de daño fue propuesto originalmente a partir de datos experimentales de pruebas de muros de mampostería no reforzada realizadas en China (Kwok y Ang 1987). En el estudio descrito en detalle por Juárez (2007) este índice se valora para nuestra modalidad de mampostería combinada y confinada. De acuerdo con la ecuación 6 , el daño estructural es función de los parámetros de respuesta $u_{m}$ y $\int d E$ y de los parámetros estructurales $u_{f}, q_{u}$ y $\varepsilon$.

Debido a falta de resultados experimentales suficientes para la modalidad de mampostería en estudio, se valoró este índice inicialmente determinando las variables estructurales en función de los pocos datos disponibles y de valores propuestos por Kwok y Ang. Como se precisa en Juárez (2007), se tomó $q_{u}$ $=5.1282$ ton y $u_{u}=12.63 \mathrm{~mm}$, correspondientes a una distorsión de 0.006 para el muro MCC-1. Considerando que el desplazamiento de falla $\left(u_{f}\right)$ es múltiplo del desplazamiento último $\left(u_{u}\right)$ y que Kwok y Ang (1987) disponían de una base de datos más extensa, se tomó su propuesta de calcular $u_{f}=\alpha u_{u}$, donde $\alpha=1.47$ y $\varepsilon=0.075$, valores propuestos por Kwok y Ang, por lo que entonces $u_{f}=18.6 \mathrm{~mm}$. Utilizando estos parámetros, se trazaron las curvas de índice de daño de Kwok y Ang vs distorsión para los muros MCC-1 y MCC-2, mismas que se presentan en la figura 26a.

El intervalo de valores para el índice de daño propuesto por Kwok y Ang (1987) se indica en la tabla 11. De acuerdo con la tabla 11 y los resultados presentados en la figura 26a para los dos muros, se observa que para una distorsión de 0.002 el daño de la estructura es reparable, coincidiendo con otros 
ensayes previos en el Cenapred, que el mayor daño de una estructura se da para distorsiones de 0.002 a 0.006, puntualizando el hecho que si un muro incursiona en distorsiones mayores a 0.006, el daño es irreparable y con tendencia irreversible de colapso.

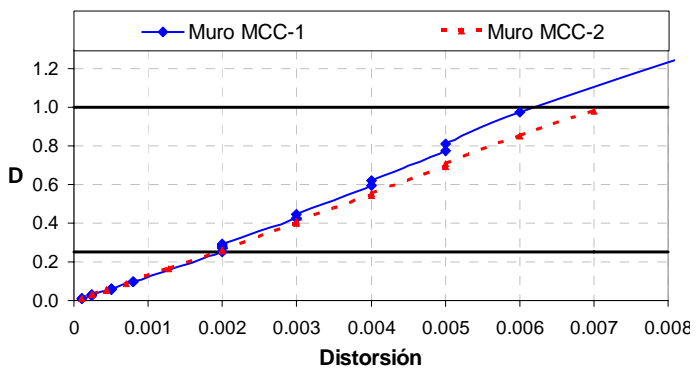

a) Valoración inicial

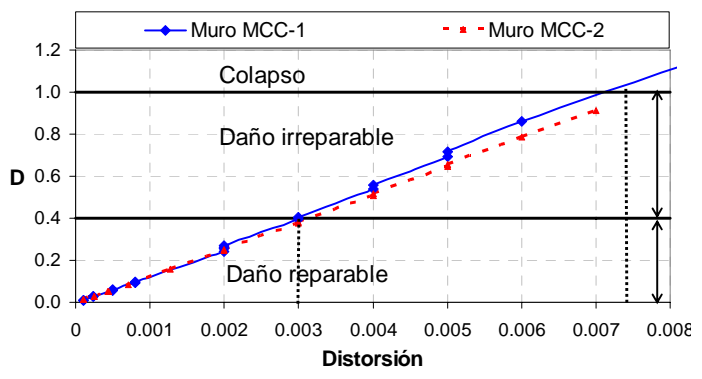

b) Ajuste propuesto

Figura 26. Índice de daño de Kwok y Ang $(D)$ vs distorsión para los muros MCC-1 y MCC-2

Tabla 11. Valores propuestos por Kwok y Ang para su índice de daño para muros no reforzados

\begin{tabular}{ccccc}
\hline & No existe daño & Daño reparable & Daño severo & Colapso inminente \\
\hline$D$ & 0 & 0 a 0.25 & 0.25 a 1.0 & $\geq 1.0$ \\
\hline
\end{tabular}

Posteriormente, se hizo un análisis más fino proponiendo un ajuste a este índice con base en el extenso análisis de los resultados experimentales, confrontando daño observado con parámetros definidos numéricamente y que sirvieron para definir que la distorsión a la que los muros de mampostería combinada y confinada experimenta un deterioro que es reparable es 0.003 (Juárez 2007), como se sintetiza secciones adelante. Con la salvedad de contar sólo con dos especimenes de este tipo de mampostería, se propone un ajuste al índice de Kwok y Ang para relacionar la respuesta de la estructura con el nivel de daño esperado, de manera que el daño reparable corresponda al observado para la distorsión de 0.003. De la curva inicial (figura 26a) se aprecia que el índice está cercano a un valor $D=0.4$, por lo que se realizó un ajuste de manera que dicho valor fuera la frontera entre daño reparable e irreparable. La metodología de los ajustes se explica con detalle en Juárez (2007), pero el parámetro ajustado obtenido es $\varepsilon=0.046$. La curva ajustada se presenta en la figura 26b, donde se definen gráficamente las fronteras propuestas para el índice para la modalidad de mampostería en estudio.

\section{Criterios de aceptación de las NTCM-2004 para resistir sismos}

En el Apéndice A de las NTCM-2004 se establecen los criterios de aceptación que se deben cumplir para que una modalidad de mampostería se considere apta para resistir cargas laterales por sismos. Los muros MCC-1 y MCC-2 se evaluaron con todo rigor conforme a estos lineamientos, como se reporta con detalle en Juárez (2007), llegando a la conclusión que el sistema estructural de muros de mampostería combinada y confinada estudiado cumple con los criterios establecidos por las NTCM-2004 para su uso en zonas sísmicas. Cabe señalar nuevamente, que los muros en estudio, se unieron con un mortero tipo I, muy distinto al que se utiliza en autoconstrucción, por lo que es urgente valorar el desempeño de muros de mampostería combinada y confinada unida con los morteros utilizados en autoconstrucción, en lo cual este equipo de trabajo está trabajando actualmente. 


\section{Propuesta de distorsión de diseño}

De acuerdo con las NTCM-2004, la distorsión permisible para muros de mampostería confinada es de:

0.0025

en muros de carga de mampostería confinada de piezas macizas (utilizando un tipo de pieza en su construcción).

en muros de carga de mampostería confinada de piezas macizas con refuerzo horizontal o mallas.

El grado de daño en la mampostería está asociado al deterioro del muro debido al agrietamiento que se presenta cada vez que se le somete a ciclos de carga. El daño en una estructura se va acumulando en cada evento sísmico sucesivo y la capacidad del sistema disminuye seriamente. Las decisiones concernientes al nivel de daño en una estructura de mampostería que es aconsejable aceptar se manejan mediante distorsiones permisibles de entrepiso. Esta distorsión está asociada a su vez, al agrietamiento que se va presentando en cada incursión de carga.

En este estudio, se propone un valor de distorsión aceptable de acuerdo con el desempeño observado en los muros de mampostería confinada y combinada (utilizando dos tipos de pieza en su construcción), sometidos a cargas laterales reversibles del tipo sísmico. La distorsión que puede permitirse debe ser la menor asociada a los siguientes tres criterios: (a) aquella previa donde se presentan desconchamientos en la mampostería, (b) donde se presenta la resistencia máxima y, (c) donde se tiene una rigidez efectiva de por lo menos el $20 \%$ de la rigidez inicial. La valoración de estos tres criterios se presenta con detalle en Juárez (2007) y se resume para ambos muros en la tabla 12. La distorsión que rigió en este caso es aquella previa al desconchamiento de las piezas, que para el caso del muro MCC-1 también coincide con la asociada a una rigidez efectiva de $20 \%$ la rigidez inicial. Este nivel de distorsión de diseño propuesto permite que la estructura sea reparable, ya que a ese nivel de daño aun las grietas no han penetrado en su totalidad en las esquinas de los castillos, y el desconchamiento aún no ha iniciado. En la figura 27 se proporcionan detalles del daño observado a este nivel de distorsión propuesto para diseño.

Tabla 12. Definición de la distorsión de diseño de muros de mampostería combinada

\begin{tabular}{lcc}
\multicolumn{1}{c}{ Criterio } & $\begin{array}{c}\text { Distorsión } \\
\text { Muro MCC-1 }\end{array}$ & $\begin{array}{c}\text { Distorsión } \\
\text { Muro MCC-2 }\end{array}$ \\
\hline $\begin{array}{l}\text { a) Distorsión previa a desconchamientos de las piezas } \\
\text { b) Distorsión asociada a la resistencia máxima }\end{array}$ & 0.003 & 0.003 \\
c) Distorsión a una rigidez efectiva de por lo menos 20\% la inicial & 0.0048 & 0.0044 \\
\hline
\end{tabular}

Figura 27. Daño observado para la distorsión de 0.003 propuesta para diseño 


\section{Patrones finales de agrietamiento}

Los patrones finales de agrietamiento se presentan en la figura 28, donde se aprecia que el modo de falla es típico de cortante. Cabe señalar que el muro MCC-1 se pudo llevar a la falla en ambas direcciones (figura 28a), mientras que en el muro MCC-2 esto no fue posible debido a que el gato hidráulico empleado presentó durante la prueba una deficiencia en poder incrementar la fuerza reversible aplicada ("jale”), por lo que en una dirección (la de “jale”) sólo se pudo llevar al espécimen a una distorsión de 0.004.

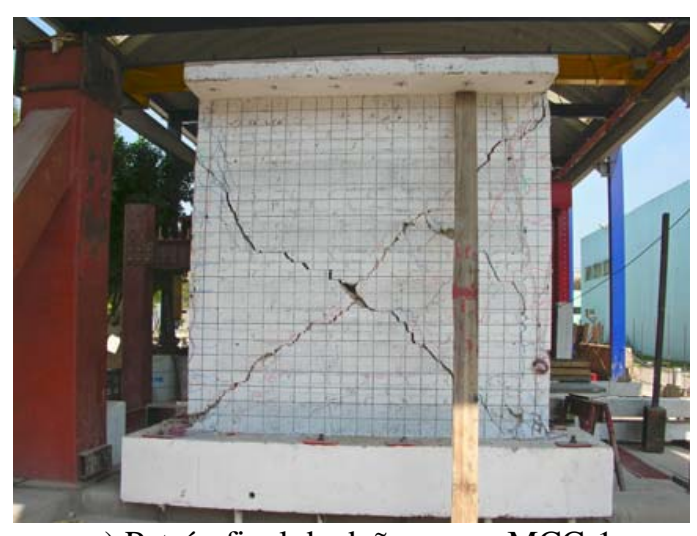

a) Patrón final de daño, muro MCC-1

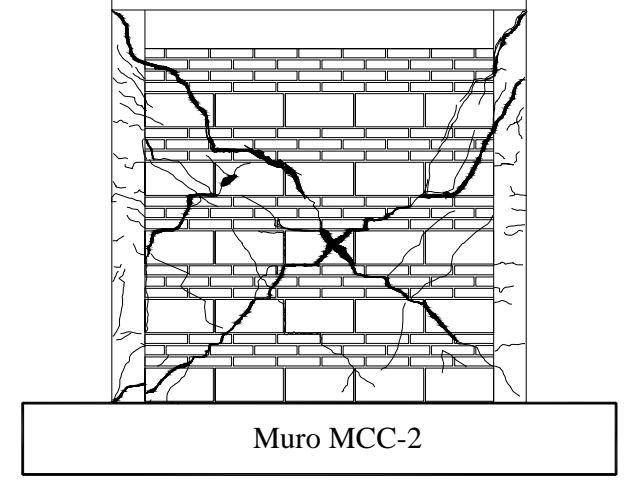

b) Patrón final de daño, muro MCC-2

Figura 27. Patrones finales de agrietamiento, muros MCC-1 y MCC-2

\section{COMPARACIÓN CON ENSAYES DE MUROS DE MAMPOSTERÍA CONFINADA}

Se realizó una recopilación de información acerca de especimenes similares ensayados de forma cuasiestática en el Cenapred, con objeto de hacer una comparación de sus resultados experimentales con los obtenidos en este estudio. La comparación fue principalmente en: lazos de histéresis, envolventes, cargas máximas, distorsión, rigidez inicial y patrón de agrietamiento, aunque también se revisaron las propiedades de los materiales utilizados en los diferentes especimenes. Cabe señalar que aunque en el Cenapred se ha realizado mucha investigación en cuanto a mampostería confinada, a nivel de muros confinados sin refuerzo horizontal, la información encontrada es escasa. La comparación se realiza con el modelo M-0-E6 (figura 28), fabricado y ensayado en el Cenapred, y que ha servido como referencia de comparación con varios muros de mampostería con refuerzo interior (horizontal y vertical).

Con respecto a la geometría, se aprecia de comparar las figuras 12 con la 28a que el muro M-0-E6 es ligeramente más ancho y alto $(2.5 \mathrm{~m} v s 2.30 \mathrm{~m})$. En cuanto a propiedades mecánicas índice, para el muro M-0-E6 se reportan $f_{m}^{*}=25 \mathrm{~kg} / \mathrm{cm}^{2}, v^{*}{ }_{m}=3.0 \mathrm{~kg} / \mathrm{cm}^{2}$ y $G_{m}=4,850 \mathrm{~kg} / \mathrm{cm}^{2}$, valores más altos que para los muros MCC-1y MCC-2 (tablas 2, 4 y 5); en cambio, el valor obtenido para $E_{m}=7,420 \mathrm{~kg} / \mathrm{cm}^{2}$ es notablemente más bajo (tabla 3).

De la comparación de patrones finales de agrietamiento (figuras 27 y 28b) se aprecia similitud y coincidencia en la falla por tensión diagonal en el tablero (en forma de cruz), presentándose los máximos desconchamientos y amplitudes de grieta en la parte central del mismo, además de las grietas que penetran a los castillos en sus extremos y algunas fisuras de flexocortante en los tercios centrales de los castillos. 


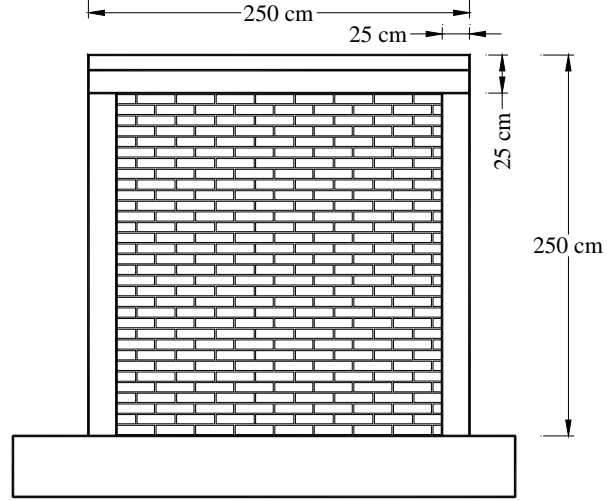

a) Geometría

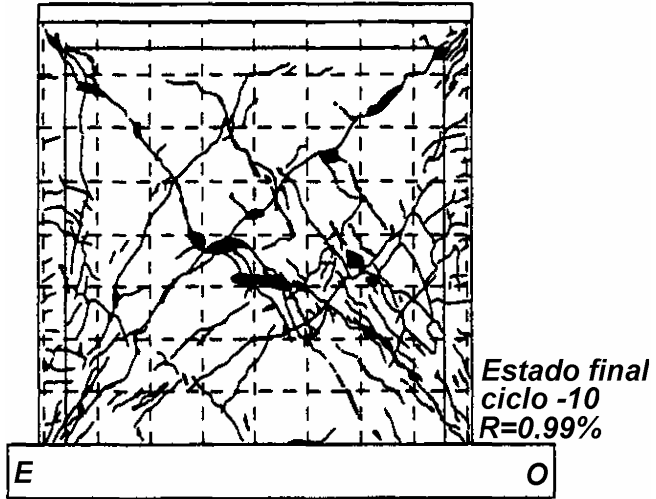

b) Patrón final de agrietamiento

Figura 28. Modelo M-0-E6 ensayado en el Cenapred (Flores y Alcocer 2001)

Las curvas de histéresis obtenidas se comparan a la misma escala en la figura 29. De la misma, se aprecia que el muro de tabique rojo recocido ensayado en el Cenapred exhibió una mayor resistencia y una mejor capacidad de deformación y, por ende, de disipación de energía. La resistencia mayor observada en el muro del Cenapred se debe a tres factores: a) un valor mayor de $v^{*}$, b) una mayor área de la sección transversal del muro y, c) mayor valor de la carga axial de compresión aplicada; en el muro del Cenapred se aplicó $\mathrm{P}=15$ ton.

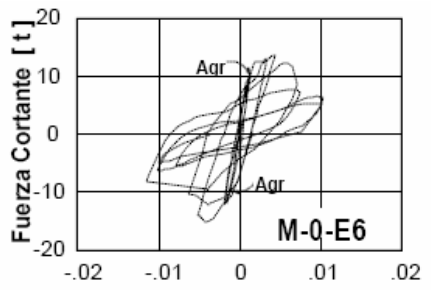

a) Muro M-0-E6

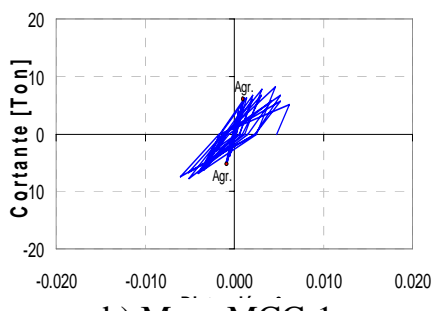

b) Muro MCC-1

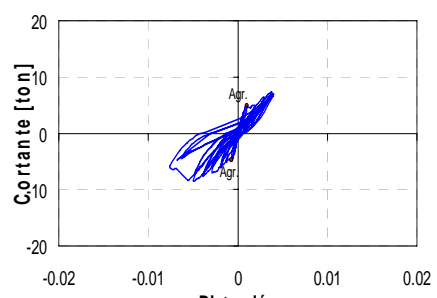

c) Muro MCC-2

Figura 29. Comparación de curvas de histéresis con el modelo ensayado en el Cenapred

En la tabla 13 se comparan algunos valores índice obtenidos del ensaye experimental. De esta comparación, se observa que tanto la mampostería combinada y confinada y la del modelo M-0-E6, se obtuvieron valores similares en cuanto a distorsiones correspondientes a cortante de agrietamiento y al cortante máximo en ambos cuadrantes, discrepando en el cortante último, el cual es notablemente menor, debido a los factores discutidos con anterioridad. En cuanto a la rigidez pico a pico inicial, la mampostería combinada tuvo mayor rigidez para el muro MCC-1 que la del modelo M-0-E6 del Cenapred, pero para el muro MCC-2 fue ligeramente inferior.

Con la finalidad de hacer una comparación más justa entre las resistencias al primer agrietamiento, se hizo una normalización de los resultados obtenidos para los muros MCC-1 y MCC-2 (tabla 13) utilizando la ecuación de diseño de las NTCM-2004 (ecuación 1). A partir de $V_{a g r}, F_{R}=1$ y tomando los valores de $A_{T}$ y $P$ de los ensayes realizados, se obtuvo que un valor equivalente $v^{*}{ }_{m}=3.15 \mathrm{~kg} / \mathrm{cm}^{2}$ para los ensayes realizados. Utilizando estos valores y los valores de $A_{T}$ y $P=15$ toneladas del muro M-0-E6 ensayado en el Cenapred, se obtiene de la ecuación 1 que la resistencia de agrietamiento esperada para los muros MCC-1 y MCC-2 sería $V_{m R}=9.2$ toneladas, bastante cercana a la reportada en la tabla 13 para el 
muro M-0-E6. Por supuesto, existen otros factores que afectan la resistencia desarrolladas en un experimento, pero lo que resulta de esta normalización es que, al parecer, las diferencias obtenidas en la resistencia al agrietamiento entre la mampostería confinada tradicional con tabique de barro rojo recocido y la combinada pudieran ser no tan grandes, quizá del orden del 5\% al 15\% en igualdad de circunstancias, lo que sería deseable verificar experimentalmente en un ensaye donde se tengan la misma geometría y carga vertical aplicada, lo que actualmente no es posible en el dispositivo experimental del laboratorio de la UAM-A.

Tabla 13. Comparación gruesa con modelos similares ensayados en el Cenapred

\begin{tabular}{|c|c|c|c|c|c|c|c|}
\hline \multirow[t]{2}{*}{ Muro } & \multirow{2}{*}{$\begin{array}{l}\text { Piezas } \\
\text { de } \\
\text { mampostería } \\
\text { utilizadas }\end{array}$} & \multicolumn{2}{|c|}{$\begin{array}{c}\text { Rigidez } \\
\text { experimental } \\
\text { (ton-mm) }\end{array}$} & \multirow[t]{2}{*}{$\begin{array}{c}V_{\text {agr }}^{-} \text {[ton] } \\
\text { (Distorsión) }\end{array}$} & \multirow[t]{2}{*}{$\begin{array}{c}V_{\max }^{+}[\text {ton] } \\
\text { (Distorsión) }\end{array}$} & \multirow[t]{2}{*}{$\begin{array}{c}V_{\max }{ }^{-[} \text {ton] } \\
\text { (Distorsión) }\end{array}$} & \multirow[t]{2}{*}{$\begin{array}{c}\text { Distorsión } \\
\text { Ultima }\end{array}$} \\
\hline & & $\mathrm{K}^{+}$ & $\mathrm{K}^{-}$ & & & & \\
\hline MCC-1 & $\begin{array}{c}\text { Bloques y } \\
\text { tabiques }\end{array}$ & 5.98 & 6.28 & $\begin{array}{c}5.0 \\
(0.0009)\end{array}$ & $\begin{array}{c}8.17 \\
(0.0046)\end{array}$ & $\begin{array}{c}7.75 \\
(0.005)\end{array}$ & 0.006 \\
\hline MCC-2 & $\begin{array}{c}\text { Bloques y } \\
\text { tabiques }\end{array}$ & 3.53 & 4.37 & $\begin{array}{c}5.0 \\
(0.0007)\end{array}$ & $\begin{array}{c}7.3 \\
(0.0037)\end{array}$ & $\begin{array}{c}8.4 \\
(0.005)\end{array}$ & 0.008 \\
\hline M-0-E6 ${ }^{1}$ & Tabiques & 4.81 & 4.24 & $\begin{array}{c}9.65 \\
(0.00100)\end{array}$ & $\begin{array}{c}13.65 \\
(0.00455)\end{array}$ & $\begin{array}{c}15 \\
(0.00415)\end{array}$ & 0.01 \\
\hline
\end{tabular}

${ }^{1}$ M-0-E6: modelo de control, Flores y Alcocer (2001)

\section{EVALUACIÓN DEL MODELO HISTERÉTICO DE FLORES Y ALCOCER PROPUESTO PARA MAMPOSTERÍA CONFINADA}

Flores y Alcocer (2001) proponen un modelo histerético basado en evidencia experimental de muros de mampostería confinada, definiendo una curva envolvente trilineal (figura 30). Este modelo considera degradación de rigidez y resistencia, variables que no toman en cuenta otros modelos previos propuestos para mampostería confinada (por ejemplo, Bazán 1980, Moroni et al. 1994).

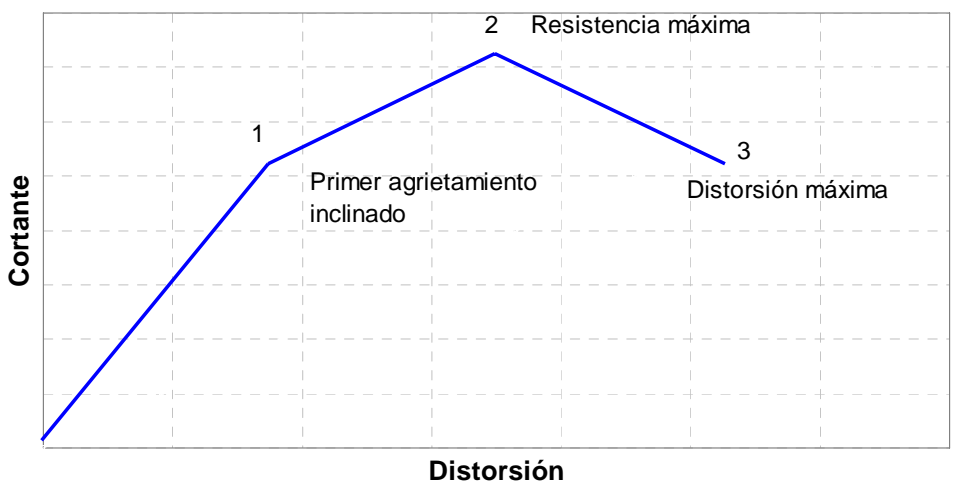

Figura 30. Envolvente trilineal del modelo propuesto por Flores y Alcocer

El modelo de Flores y Alcocer (2001) se calibró con muros de uno y dos pisos con y sin refuerzo horizontal, respuestas dinámicas de estructuras de un grado de libertad y la aplicación del modelo en estructuras de mampostería empleando registros sísmicos reales. 
Por todo lo anterior, se decidió valorar este modelo para la modalidad de mampostería combinada y confinada en estudio. Aunque el modelo de Flores y Alcocer se restringe a muros de mampostería confinada, construidos a base de tabiques de barro recocido, los autores mencionaron que esperarían comportamientos similares con materiales como bloques de concreto.

En términos generales, el modelo consiste en construir la curva histerética siguiendo una rama ascendente de cada ciclo con cierta rigidez, hasta alcanzar el primer agrietamiento, y seguir sobre la envolvente hasta la carga máxima. Al descargar, la trayectoria es una línea recta cuya pendiente depende de la distorsión máxima alcanzada hasta ese momento (figura 30).

El modelo propuesto por Flores y Alcocer involucra seis variables que dependen de la resistencia y deformación de la estructura. Las variables que considera son:

$K_{0} \quad$ Rigidez inicial de la estructura

$\underline{V}_{\text {agr }} \quad$ Carga correspondiente al agrietamiento de la mampostería

$V_{\max } \quad$ Carga máxima

$V_{u} \quad$ Carga última

$H \quad$ Altura del espécimen

$\theta_{V \max } \quad$ Distorsión asociada a la carga máxima

$\theta_{u} \quad$ Distorsión asociada a la carga ultima

Las diferencias entre los muros ensayados en el Cenapred y los aquí reportados en cuanto a geometría, materiales y valor de la carga vertical aplicada, se han discutido previamente. Para la aplicación del modelo de Flores y Alcocer se utilizaron los resultados obtenidos de los ensayes de los muros MCC-1 y MCC-2, indicados en negritas en la tabla 14. Para la resistencia última y su distorsión asociada, así como la distorsión asociada a la carga máxima, se utilizan los datos que recomiendan Flores y Alcocer para mampostería confinada sin refuerzo horizontal. Estos datos se presentan en la tabla 14.

Tabla 14. Datos experimentales (absolutos) de los muros MCC-1 y MCC-2

\begin{tabular}{cccccccc}
\hline Variable & $\begin{array}{c}K_{0} \\
\text { ton-cm }\end{array}$ & $\begin{array}{c}V_{\text {agr }} \\
\text { ton }\end{array}$ & $\begin{array}{c}V_{\max } \\
\text { ton }\end{array}$ & $\begin{array}{c}V_{u} \\
\text { ton }\end{array}$ & $\begin{array}{c}H \\
\mathrm{~cm}\end{array}$ & $\theta_{V \max }$ & $\theta_{u}$ \\
\hline MCC-1 & $\mathbf{6 2 . 9}$ & $\mathbf{5 . 0}$ & $\mathbf{8 . 1 7}$ & $4.0^{1}$ & $\mathbf{2 1 0 . 5}$ & $0.003^{2}$ & $0.01^{2}$ \\
MCC-2 & $\mathbf{4 3 . 7}$ & $\mathbf{5 . 0}$ & $\mathbf{8 . 3 5}$ & $4.0^{1}$ & $\mathbf{2 2 0 . 0}$ & $0.003^{2}$ & $0.01^{2}$ \\
\hline
\end{tabular}

En la figura 31 se comparan las gráficas de histéresis experimentales para los especimenes MCC-1, MCC-2 y las calculadas con el modelo propuesto por Flores y Alcocer. Como se observa, dicho modelo analítico tiene la capacidad de representar de forma muy razonable la respuesta histerética obtenida experimentalmente, inclusive para el muro MCC-2, en el cual los semiciclos positivos no se completaron para distorsiones mayores a 0.004. Esto es notable, pues el modelo de Flores y Alcocer se desarrolló empleando una base experimental de ensayes de mampostería confinada elaborada con tabiques de barro recocido exclusivamente.

Para mejorar la aproximación, se ajustaron todos los parámetros que involucra el modelo, con base en los resultados experimentales, para lo cual se calculó el promedio de los valores obtenidos en ambos cuadrantes. Los parámetros se resumen en la tabla 15. Las curvas analíticas obtenidas con estos ajustes se comparan con las experimentales en las figuras 32 y 33, donde se aprecia claramente una mejor 
correlación en las curvas de histéresis del muro MCC-2 (figura 32) y en las curvas envolventes (figura 33).

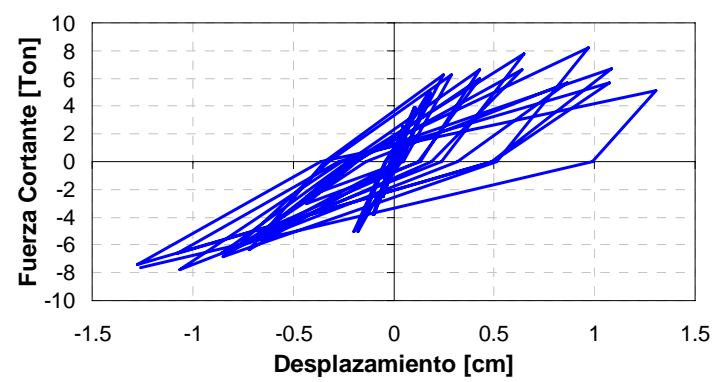

a) Curva experimental modelo MCC-1

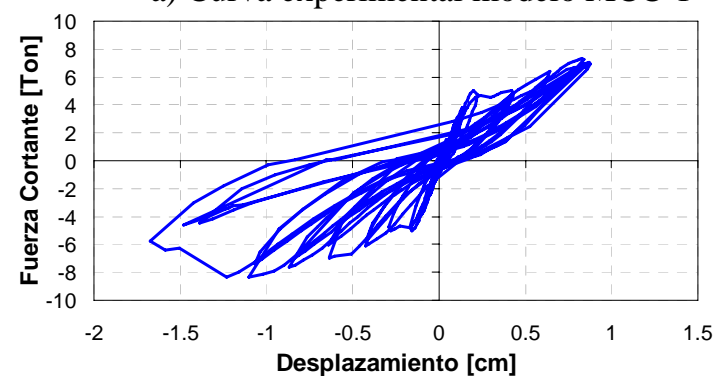

c) Curva experimental modelo MCC-2

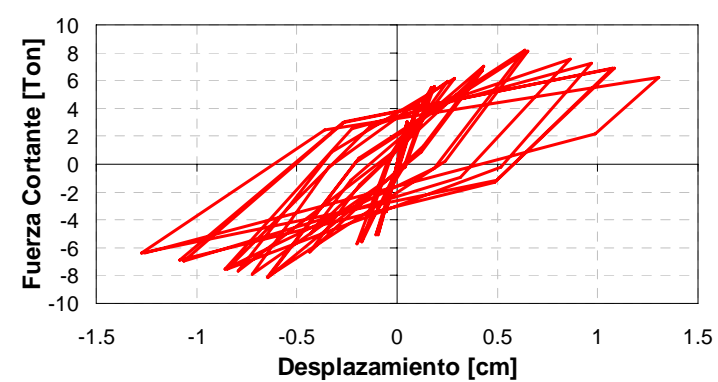

b) Curva analítica modelo MCC-1

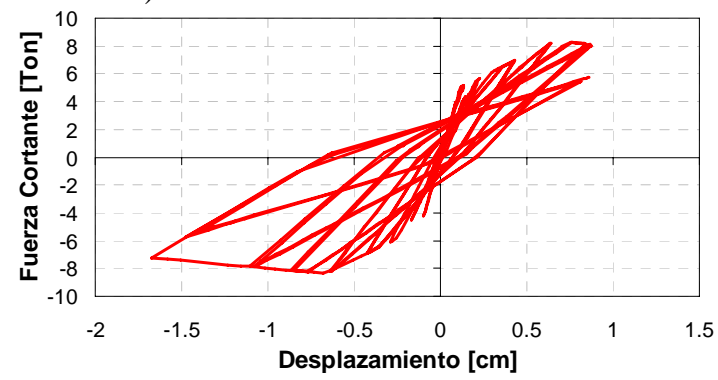

d) Curva analítica modelo MCC-2

Figura 31. Curvas de histéresis experimental vs calculada analíticamente con el modelo de Flores y Alcocer

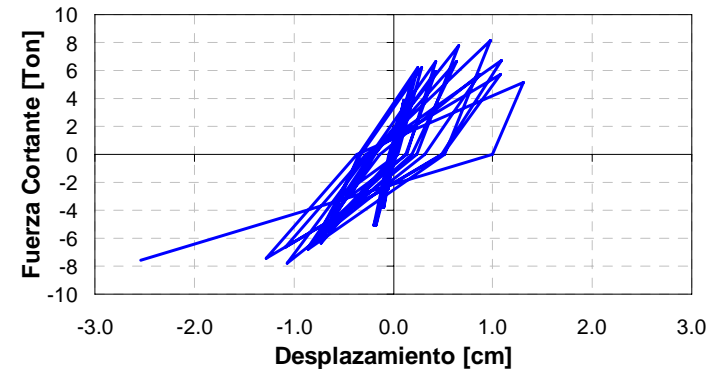

a) Curva experimental modelo MCC-1

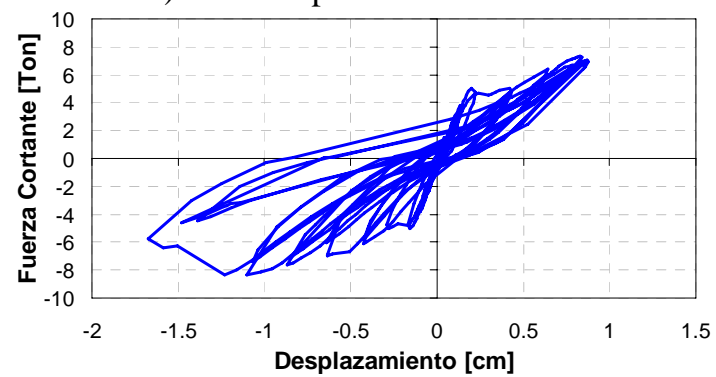

c) Curva experimental modelo MCC-2

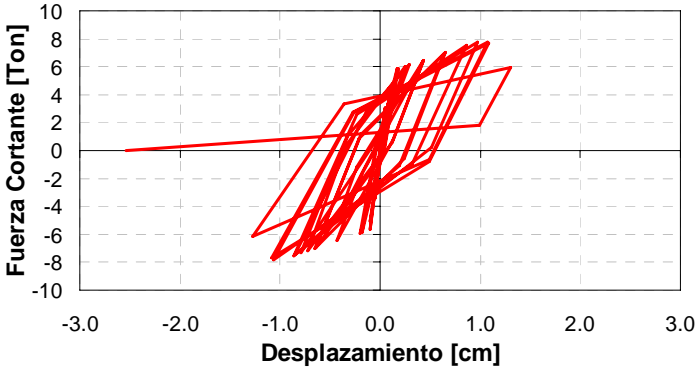

b) Curva analítica modelo MCC-1

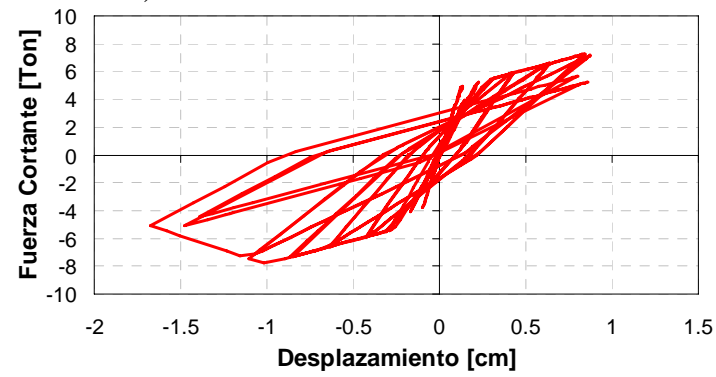

d) Curva analítica modelo MCC-2

Figura 32. Curvas de histéresis experimental vs calculada analíticamente con el modelo de Flores y Alcocer, después de los ajustes experimentales 
Arturo Tena Colunga, Artemio Juárez Ángeles y Víctor Hugo Salinas Vallejo

Tabla 15. Datos experimentales (promedio) de los muros MCC-1 y MCC-2

\begin{tabular}{ccccccccc}
\hline Variable & $\begin{array}{c}K_{0} \\
\text { ton-cm }\end{array}$ & $\begin{array}{c}V_{\text {agr }} \\
\text { ton }\end{array}$ & $\begin{array}{c}V_{\max } \\
\text { ton }\end{array}$ & $\begin{array}{c}V_{u} \\
\text { ton }\end{array}$ & $\begin{array}{c}H \\
\mathrm{~cm}\end{array}$ & $\theta_{V \max }$ & $\theta_{u}$ \\
\cline { 1 - 7 } MCC-1 & 61.5 & 5.65 & 7.97 & 6.28 & $210.5^{1}$ & 0.0048 & 0.006 \\
MCC-2 & 39.5 & 4.9 & 7.83 & 5.66 & $220.0^{1}$ & 0.0046 & 0.007 \\
\hline
\end{tabular}

${ }^{1}$ Único parámetro que no se modificó

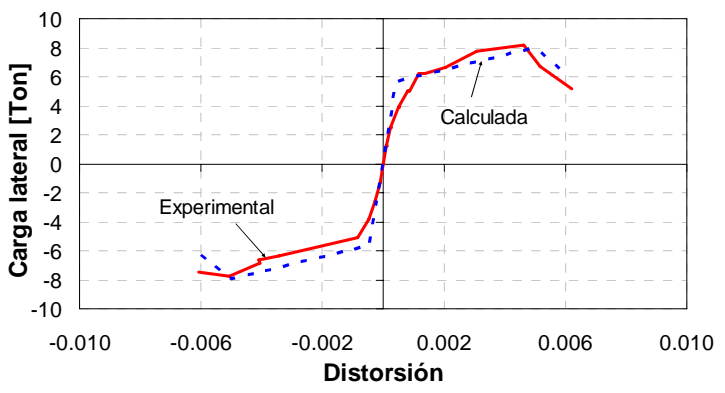

a) Envolvente experimental vs propuesta para el modelo histerético, muro MCC-1

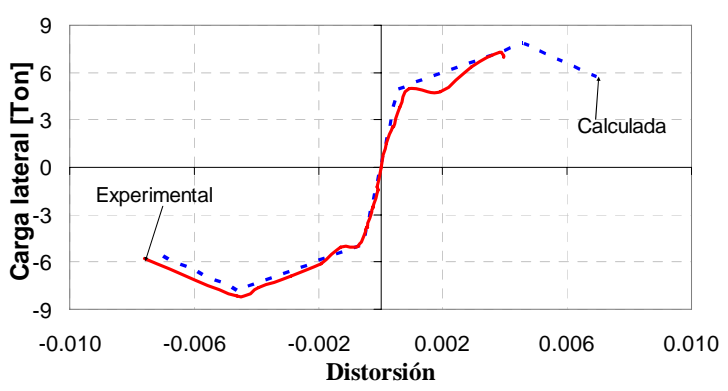

b) Envolvente experimental vs propuesta para el modelo histerético, muro MCC-2

Figura 33. Envolvente experimental vs calculada analíticamente con el modelo de Flores y Alcocer, después de los ajustes experimentales

Finalmente, se consideró interesante comparar la predicción de la respuesta de estos muros si, $a$ priori, se utilizan sus propiedades mecánicas $\left(v^{*}, E_{m}\right.$ y $\left.G_{m}\right)$ determinadas con base en los ensayes simples de muretes y pilas, además de las propiedades de su sección transversal y dimensiones, suponiendo que esto se realiza antes del ensaye experimental del muro en sí. Dado que en los ensayes de los muretes del arreglo 1, se apreciaron deslizamientos que pudieran redundar, en opinión de los autores, en resistencias menos representativas, se decidió realizar esta comparación sólo con las propiedades mecánicas reportadas para el arreglo 2. Desde esta óptica, los datos para alimentar al modelo de Flores y Alcocer son los reportados en la tabla 16, marcándose con negritas los valores calculados, como se detalla en Juárez (2007).

Tabla 16. Datos teóricos de muros de mampostería combinada y confinada

\begin{tabular}{|c|c|c|c|c|c|c|c|}
\hline Variable & $\begin{array}{c}K_{0} \\
\text { ton-cm }\end{array}$ & $\begin{array}{l}V_{\text {agr }} \\
\text { ton }\end{array}$ & $\begin{array}{l}V_{\max } \\
{\left[1.25 V_{\text {agr }}\right]} \\
\text { ton }\end{array}$ & $\begin{array}{c}V_{u} \\
{\left[0.8 V_{\text {agr }}\right]} \\
\text { ton }\end{array}$ & $\begin{array}{c}H \\
\text { [real] } \\
\text { cm }\end{array}$ & $\theta_{V \max }$ & $\theta_{u}$ \\
\hline $\begin{array}{c}\text { Mampostería combinada y } \\
\text { confinada con } v_{m}^{*}\end{array}$ & 49.1 & 4.2 & 5.26 & 3.36 & 220 & 0.003 & 0.01 \\
\hline $\begin{array}{l}\text { Mampostería combinada y } \\
\text { confinada con } \nabla_{m}\end{array}$ & 49.1 & 5.9 & 7.41 & 4.75 & 220 & 0.003 & 0.01 \\
\hline
\end{tabular}

Las curvas calculadas con los valores asociados con $v_{m}^{*}$ se muestran con línea discontinua en la figura 34, donde se comparan con la experimental. Se aprecia que se subestima la resistencia a cortante y que se predice un comportamiento cíclico más estable que el obtenido experimentalmente. 


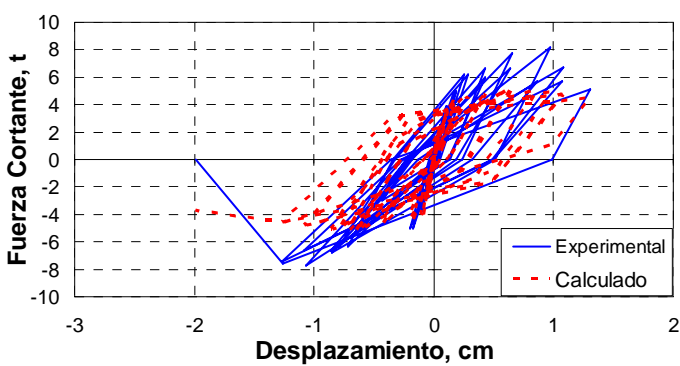

a) Curva experimental vs calculada, Muro MCC-1

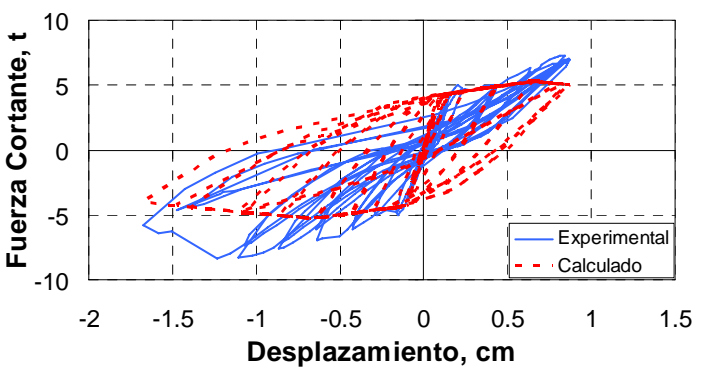

b) Curva experimental vs calculada, Muro MCC-2

Figura 34. Envolvente experimental vs calculada analíticamente con el modelo de Flores y Alcocer, utilizando los valores de ensayes de pilas y muretes, para $v_{m}^{*}$

Igualmente, las curvas calculadas con los valores asociados con $v_{m}$ se muestran con línea discontinua en la figura 35, donde se comparan con la experimental. En este caso, se aprecia que la estimación de la respuesta con el modelo analítico está más apegada a la realidad experimentalmente registrada.

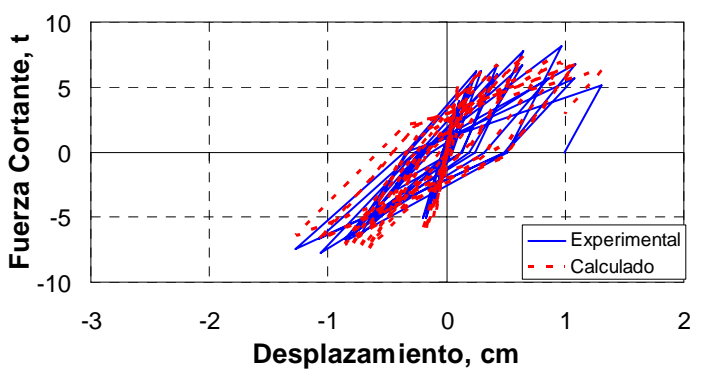

a) Curva experimental vs calculada, Muro MCC-1

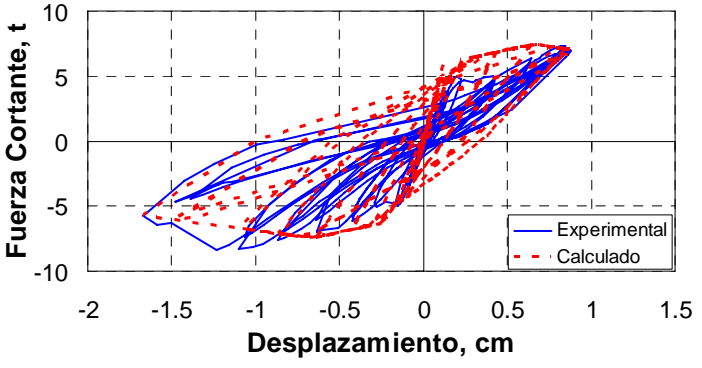

b) Curva experimental vs calculada, Muro MCC-2

Figura 35. Envolvente experimental vs calculada analíticamente con el modelo de Flores y Alcocer, utilizando los valores de ensayes de pilas y muretes, para $\bar{\nabla}_{m}$

\section{COMENTARIOS FINALES}

Se presentaron los resultados más relevantes de todo un protocolo de pruebas experimentales que culminaron con el ensaye cíclico de dos muros de la mampostería combinada y confinada que se emplea actualmente en zonas sísmicas de México, siendo éstos los primeros en realizarse en el mundo para esta modalidad de mampostería. Los ensayes se realizaron conforme al protocolo para ensayes cíclicos establecido en el Apéndice A de las Normas Técnicas para Diseño y Construcción de Estructuras de Mampostería (NTCM-2004), por lo que los especimenes se sujetaron a ciclos repetidos, primero ante cargas dadas (hasta la carga de agrietamiento), y después a deformaciones (distorsiones angulares) impuestas de manera creciente.

Se valoraron tanto los mecanismos resistentes, como las características de deformación de esta modalidad de mampostería, evaluando parámetros útiles para fines de análisis y diseño, como son la estimación de cargas resistentes a partir de propiedades índice, la misma definición de las propiedades índice de cada material utilizado y del conjunto, definición de distorsiones índice y sus índices de daño 
asociados, características de degradación de rigidez y de disipación de energía, entre muchos otros aspectos relevantes. Además, se hicieron comparaciones generales con respecto a los resultados del muro M-0-E6, un muro confinado de tabique rojo recocido ensayado en el Cenapred durante la década de los años noventa, y se evaluó el modelo histerético propuesto por Flores y Alcocer (2001) para muros de mampostería confinada.

De acuerdo con el nivel de daño observado durante los ensayes, se propone una distorsión de diseño igual a $\theta=0.003$ para muros con uso estructural, que permitiría que un muro de mampostería combinada y confinada tenga un nivel de daño que pueda ser reparable de manera económica, al mismo tiempo de garantizar su estabilidad, proporcionando de esta manera seguridad a las personas que puedan habitar las estructura que los contenga, sobre todo si el sistema estructural ante cargas verticales y laterales es con base en muros de este tipo. En cambio, si se utiliza como muro diafragma, el límite actual de $\theta=0.006$ propuesto por las NTCM-2004 parece ser razonable también para esta modalidad de mampostería.

De los resultados experimentales se concluye que esta modalidad de mampostería, conforme a las NTCM-2004, es apta para resistir cargas laterales por sismo, si se emplea mortero tipo I en su pega y se confina conforme a los lineamientos de las normas de referencia. Sin embargo, como esta modalidad se está construyendo con morteros de autoconstrucción muy distintos al mortero tipo I, se debe también valorar experimentalmente la pertinencia ante sismos de esta modalidad de muro cuando se emplean morteros de autoconstrucción, en lo que se encuentra trabajando actualmente este equipo de investigación.

De la investigación que se presenta, también se desprende la duda de qué tan representativo es el ensaye de muretes a compresión diagonal para definir la propiedad índice $v^{*}$ para esta modalidad de mampostería, dada la gran diferencia en los resultados que se obtuvieron en los ensayes. Esto deberá seguirse valorando con más ensayes experimentales, antes de llegar a una conclusión contundente al respecto.

La mampostería combinada y confinada presenta similitudes con respecto a los muros confinados de tabique rojo recocido en cuanto a patrones de agrietamiento, distorsión de agrietamiento y distorsión de diseño, aunque su resistencia a cortante máxima, su capacidad de deformación última y sus propiedades índice son inferiores a la de los muros construidos exclusivamente con tabiques de barro recocido.

Las disminuciones en las propiedades globales de la mampostería combinada parecen estar relacionadas con las pobres propiedades mecánicas de los bloques empleados, por lo que también sería importante valorar esta modalidad con bloques producidos con un mayor control de calidad. Además, deben valorarse cómo influyen en la resistencia y capacidad de deformación otros aparejos de bloques y ladrillos que actualmente se utilizan, como se documenta en este estudio.

De la evaluación del modelo histerético propuesto por Flores y Alcocer para muros de mampostería confinada con base en tabique rojo recocido se llega a la conclusión que también puede representar razonablemente la respuesta histerética de los muros de mampostería combinada y confinada ensayados en este estudio. Si al modelo se le alimenta con variables determinadas a partir de los ensayes experimentales, éste es bastante robusto y predice razonablemente los lazos histéresis y la envolvente asociada a éstos. En cambio, si el modelo se alimenta con los datos provenientes de propiedades índice determinadas del ensaye de muretes y pilas, las cuales ya se encuentran reducidas por el coeficiente de variación que toma en cuenta las incertidumbres en las propiedades de los materiales debidos a procesos de fabricación y calidad de mano de obra, como debiera esperarse, se tiende a subestimar las resistencias máxima y de agrietamiento, y a predecir curvas de histéresis y envolventes algo distintas a las 
experimentales, aunque la predicción de los niveles de deformación que pueden desarrollarse es más cercana que para los niveles de resistencia desarrollados.

Asimismo, se valoró el índice de Kwok y Ang, que fue desarrollado y calibrado a partir de ensayes experimentales hechos en China de muros mampostería no reforzada con base en ladrillos. En este trabajo se propone un ajuste preliminar a este índice para mampostería combinada y confinada, reconociendo que los resultados de dos muros son insuficientes para darle robustez final al ajuste propuesto. El ajuste hecho define que el intervalo de daño reparable de este índice debe estar entre 0.0 y 0.4 , tomando como referencia la distorsión de diseño propuesta para el sistema que es $\theta=0.003$. Fue necesario hacer un ajuste preliminar de la constante $\varepsilon$ del índice, basándonos sólo en los resultados experimentales de los dos muros ensayados. Cabe señalar que esta es una propuesta preliminar que deberá pulirse conforme se cuente con un mayor número de resultados experimentales de muros de estas características.

\section{AGRADECIMIENTOS}

Este estudio no hubiera sido posible sin la colaboración de innumerables colegas y alumnos de la Universidad Autónoma Metropolitana Azcapotzalco. Se agradece, por tanto, al Técnico Académico Leopoldo Quiroz, el Técnico Rubén Barrera, el Sr. José Luis Caballero y la Sra. Silvia Ledezma por su invaluable apoyo en el desarrollo del trabajo experimental, al M. en I. Hans I. Archundia por su apoyo en la planeación de los ensayes experimentales, de la instrumentación y en la adquisición de datos, y a los entusiastas alumnos de la carrera de ingeniería civil que han participando de diversas maneras en el desarrollo de sus talleres de ingeniería civil: César Carpio, José Manuel Alonso, Misael Bahena, Daniel Miranda, Sergio López, Eder Gudiño, Rosaura Ramírez, Efraín Joaquín Diego, Marco Antonio Rico, René Espinoza, Richard Véliz, Roberto Moreno, Elías Josué Moral y Gerardo Ibarra. Finalmente, se agradecen las valiosas opiniones y experiencias que compartió con nosotros el M. en I. Leonardo Flores del Cenapred.

\section{REFERENCIAS}

Bazán, T E (1980), “Muros de mampostería ante cargas laterales. Estudios analíticos”, Tesis doctoral, División de Estudios de Posgrado, Facultad de Ingeniería, UNAM, septiembre.

Chopra, A K (1995), Dynamics of Structures, primera edición, Prentice Hall, pp. 91-103.

Flores, L y S M Alcocer (2001), "Estudio analítico de estructuras de mampostería confinada”, Informe Técnico, Coordinación de Investigación, Área de Ingeniería Estructural y Geotecnia, Cenapred, ISBN: 970-628-606-3.

Hibbeler, R C (1998), Mecánica de materiales, tercera edición, Prentice Hall, México, pp 85-99.

Juárez, A (2007), "Mecanismos de resistencia y de deformación de muros de mampostería combinada y confinada”, Tesis de Maestría, División de Ciencias Básicas e Ingeniería, Universidad Autónoma Metropolitana Azcapotzalco (en prensa).

Kwok, Y-H y A H-S Ang (1987), "Seismic damage analysis and design of unreinforced masonry buildings”, Structural Research Series No. 536, University of Illinois at Urbana-Champaign, junio.

Mc Nary, W S y D P Abrams (1985), "Mechanics of masonry in compression”, ASCE Journal of Structural Engineering, Vol. 111, No. 4, pp. 857-870, abril. 
Moroni, M O, M Astroza y S Tavonatti (1994), "Nonlinear models for shear failure in confined masonry walls”, The Masonry Society Journal, Vol. 12, No. 2, pp 72-77, febrero.

NMX-C-404-ONNCCE (1997), "Industria de la construcción-bloques, tabiques o ladrillos y tabicones para uso estructural-especificaciones y métodos de prueba”, Organismo Nacional de Normalización y Certificación de la Construcción y Edificación, México, 9 pp.

NTCM-2004 (2004), "Normas Técnicas Complementarias para Diseño y Construcción de Estructuras de Mampostería”, Gaceta Oficial del Distrito Federal, octubre.

PROYNMX-S/N-ONNCCE-2005 (2005), "Determinación de la resistencia a compresión diagonal y rigidez a cortante de muretes y la resistencia a compresión y módulo de elasticidad de pilas de mampostería de barro o de concreto-Método de prueba”, Organismo Nacional de Normalización y Certificación de la Construcción y Edificación, México, 12 pp.

Salinas, V H (2005), "Comportamiento de muros de mampostería combinada y confinada ante cargas laterales”, Proyectos Terminales I y II, Departamento de Materiales, Universidad Autónoma Metropolitana, septiembre.

Schneider R R y W H Dickey (1993), Reinforced Masonry Design, tercera edición, Prentice Hall.

Tena, A (1994) "Diseño de estructuras de mampostería”, Apuntes del curso dictado de 1993 a la fecha en la División de Estudios de Posgrado de la Facultad de Ingeniería, UNAM y en la UAM Azcapotzalco, México. 